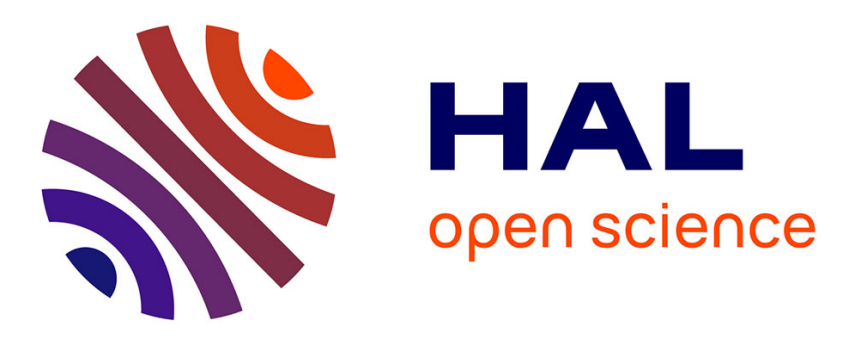

\title{
Nonlinear self-sustained structures and fronts in spatially developing wake flows
}

Benoît Pier, Patrick Huerre

\section{To cite this version:}

Benoît Pier, Patrick Huerre. Nonlinear self-sustained structures and fronts in spatially developing wake flows. Journal of Fluid Mechanics, 2001, 435, pp.145-174. 10.1017/S0022112001003652 . hal00119721

\section{HAL Id: hal-00119721 \\ https://hal.science/hal-00119721}

Submitted on 11 Dec 2006

HAL is a multi-disciplinary open access archive for the deposit and dissemination of scientific research documents, whether they are published or not. The documents may come from teaching and research institutions in France or abroad, or from public or private research centers.
L'archive ouverte pluridisciplinaire HAL, est destinée au dépôt et à la diffusion de documents scientifiques de niveau recherche, publiés ou non, émanant des établissements d'enseignement et de recherche français ou étrangers, des laboratoires publics ou privés. 


\title{
Nonlinear self-sustained structures and fronts in spatially developing wake flows
}

\author{
By BENOÎT PIER† AND PATRICK HUERRE \\ Laboratoire d'Hydrodynamique (LadHyX), CNRS - École polytechnique, \\ F-91128 Palaiseau cedex, France
}

(Received 29 February 2000 and in revised form 25 September 2000)

A family of slowly spatially developing wakes with variable pressure gradient is numerically demonstrated to sustain a synchronized finite-amplitude vortex street tuned at a well-defined frequency. This oscillating state is shown to be described by a steep global mode exhibiting a sharp Dee-Langer-type front at the streamwise station of marginal absolute instability. The front acts as a wavemaker which sends out nonlinear travelling waves in the downstream direction, the global frequency being imposed by the real absolute frequency prevailing at the front station. The nonlinear travelling waves are determined to be governed by the local nonlinear dispersion relation resulting from a temporal evolution problem on a local wake profile considered as parallel. Although the vortex street is fully nonlinear, its frequency is dictated by a purely linear marginal absolute instability criterion applied to the local linear dispersion relation.

\section{Introduction}

The qualitative behaviour of spatially developing free shear flows such as wakes, jets and mixing layers may be reasonably well understood within the framework of linear instability theory. In this context, one may distinguish between amplifiers, the dynamics of which are sensitive to inflow perturbations, and oscillators, which sustain intrinsic global modes tuned at a well-defined frequency. Co-flow mixing layers, constantdensity jets, and wakes below the onset of Kármán vortex shedding belong to the former class, while mixing layers with a sufficiently strong countercurrent (Strykowski \& Niccum 1991), wakes in the Kármán vortex shedding régime (Provansal, Mathis \& Boyer 1987; Triantafyllou, Triantafyllou \& Chryssostomidis 1986), and inhomogenous jets (Monkewitz et al. 1990) belong to the latter class. The amplifier or oscillator behaviour is intimately related to the convective or absolute nature of the linear instability (Huerre \& Rossi 1998). Note however that absolute instability may also induce a rapid transition to turbulence as strikingly demonstrated by Lingwood (1995, 1996) for rotating disk boundary layers.

One of the central problems regarding the dynamics of flow oscillators is concerned with the prediction of the overall frequency and associated spatial structure, hereafter referred to as the global mode. For real flows, governed by the Navier-Stokes equations, this question has until now been studied solely in the framework of linear theory (Monkewitz, Huerre \& Chomaz 1993). The objective of the present analysis is to address the same issue in the fully nonlinear régime.

$\dagger$ Present address: Department of Applied Mathematics and Theoretical Physics, University of Cambridge, Silver Street, Cambridge CB3 9EW, UK. 
Koch (1985) appears to be the first to have related the onset of global oscillations in wakes to the existence of an absolutely unstable region immediately downstream of the obstacle. Further progress in our understanding of frequency selection mechanisms has typically proceeded in two distinct steps. The theoretical formulation is first established for relatively simple one-dimensional evolution models such as the celebrated complex Ginzburg-Landau (CGL) equation (Cross \& Hohenberg 1993). It is then adapted and generalized to real flows governed by the Navier-Stokes equations. This line of thought has been fully implemented in the linear approximation. Linear global modes pertaining to the CGL equation with varying coefficients on an infinite interval have been analysed by Chomaz, Huerre \& Redekopp (1991), and Le Dizès et al. (1996). The formulation essentially relies on the assumption of slow spatial variations characterized by the slow space variable $X=\epsilon x$, where $\epsilon \ll 1$ is a small nonuniformity parameter. A complex local absolute frequency $\omega_{0}(X)$ may then be defined in classical fashion (Briggs 1964, Bers 1983, Huerre \& Monkewitz 1990) by imposing a zero group velocity condition on the local linear dispersion relation. Under these conditions, the complex global frequency $\omega_{s}$ of self-sustained linear structures is given by the saddle point criterion

$$
\omega_{s}=\omega_{0}\left(X_{s}\right) \quad \text { with } \quad \frac{\mathrm{d} \omega_{0}}{\mathrm{~d} X}\left(X_{s}\right)=0,
$$

where it is understood that $\omega_{0}(X)$ has been analytically continued in the complex $X$ plane. Whenever $\omega_{s, i} \equiv \operatorname{Im} \omega_{s}>0$, the medium is globally unstable. Global instability then requires a finite region of local absolute instability where $\omega_{0, i}(X)>0$ for real $X$, as demonstrated by Chomaz et al. (1991) and Le Dizès et al. (1996). The same criterion (1.1) was previously derived by Soward \& Jones (1983) for oscillating states in Taylor-Couette flow between concentric spheres. As shown by Monkewitz et al. (1993) it also holds for the two-dimensional Navier-Stokes equations linearized about an arbitrary slowly streamwise developing shear flow. It should be emphasized that other frequency selection criteria have been proposed. In spatially developing flows of semi-infinite streamwise extent, say $x>0$, the global frequency is given by the absolute frequency $\omega_{0}(0)$ at the upstream boundary (Monkewitz et al. 1993; Woodley $\&$ Peake 1997; Taylor \& Peake 1999). The initial resonance principle conjectured by Monkewitz \& Nguyen (1987) is of particular interest in the present context: according to these authors, in spatially developing flows with an absolutely unstable region, the first streamwise station of non-negative absolute growth rate imposes its absolute frequency on the global oscillations.

The theoretical prediction (1.1) has been tested in direct numerical simulations. Schär \& Smith (1993) have numerically investigated the flow behind a vertical cylinder in the shallow-water wave régime. At a critical value of the Froude number, the wake is observed to undergo a transition to large-scale Kármán vortex shedding. When all nonlinear terms in the numerical code are turned off, the wake beats at a global frequency $\omega_{g} \sim 0.17+0.045$ i. Application of criterion (1.1) leads to the prediction $\omega_{g} \sim 0.19+0.040 \mathrm{i}$, which compares very favourably with the computed value. However, when all nonlinearities are restored, the observed global frequency becomes $\omega_{g} \sim 0.27$, which is noticeably different from the predicted linear value. Such a comparison clearly calls for a fully nonlinear formulation of the global mode problem. A similar comparative study has recently been undertaken by Hammond \& Redekopp (1997) in the case of the wake behind a blunt-edged plate. At a Reynolds number $25 \%$ above global onset, the numerically determined non-dimensional global frequency is found to be $\omega_{g} / 2 \pi \sim 0.1000$, whereas the saddle-point criterion (1.1) 
yields $\omega_{s, r} / 2 \pi \equiv \operatorname{Re} \omega_{s} / 2 \pi \sim 0.1006$. In view of the linear nature of the criterion, such a close agreement is somewhat surprising. It is worth noting, however, that the complex absolute frequency $\omega_{0}(X)$ has been computed on the mean velocity profile in the presence of finite-amplitude oscillations and not on the undisturbed basic flow, as in the case of Schär \& Smith (1993). Nonlinear effects are thereby partially accounted for through the Reynolds stresses which produce the mean flow.

The next step in the theoretical analysis of spatially developing flows would naturally involve a weakly nonlinear formulation in the vicinity of global mode onset. As emphasized by Le Dizès et al. (1993), this approach is fraught with difficulties. For slowly spatially developing flows the Landau constant governing the nature of the bifurcation has neither a well-defined limit nor a constant sign as $\epsilon$ goes to zero. As a result, the bifurcation keeps switching between subcritical and supercritical. In order to circumvent these technical difficulties, it seems appropriate to directly proceed to a fully nonlinear approach.

Most nonlinear studies have so far been restricted to amplitude evolution equations in one space dimension. Linear absolute/convective instability concepts have been generalized to finite-amplitude disturbances by Chomaz (1992): the propagation direction of fronts separating the basic state from the bifurcated state, as originally defined by Dee \& Langer (1983), Saarloos $(1988,1989)$ and Saarloos \& Hohenberg (1992), is found to essentially determine the absolute/convective nature of the instability in the nonlinear régime. Fully nonlinear global modes on a semi-infinite domain $(x>0)$ governed by Ginzburg-Landau-type model equations with constant coefficients have been thoroughly analysed by Couairon \& Chomaz (1996, 1997a,b). Whenever the medium is nonlinearly absolutely unstable, an upstream travelling front is pinned at the upstream boundary point and a nonlinear global mode is sustained. The corresponding analysis of the finite interval problem has been addressed by Tobias, Proctor \& Knobloch (1998) and Chomaz \& Couairon (1999). Couairon \& Chomaz (1999) have further investigated the existence of nonlinear global modes of the real Ginzburg-Landau equation with varying coefficients on the semi-infinite domain $x>0$. In this case, spatial inhomogeneities due to both a boundary point and varying bulk properties are combined. Although this model constitutes only a crude approximation of real flows, predicted scaling laws for the global mode amplitude and the position of its maximum agree surprisingly well with experimental and numerical results pertaining to the wake structure behind bluff bodies.

Fully nonlinear global modes on an infinite interval, have also been investigated for the CGL equation with varying coefficients in order to mimic the streamwise non-uniformity of spatially developing shear flows. Two distinct varieties of nonlinear objects are then possible. Soft global modes obtained by Pier \& Huerre (1996) satisfy a saddle-point frequency selection criterion applied to the local nonlinear dispersion relation governing finite-amplitude states. The corresponding extended spatial structure displays smoothly varying amplitude and wavenumber everywhere. By contrast, steep global modes, as described by Pier et al. (1998), obey a marginal stability criterion: the steep global frequency coincides with the real absolute frequency at the transition station between linear convective and absolute instability. More specifically, the steep global mode is triggered at the upstream boundary $X^{c a}$ of the absolutely unstable domain and is tuned at the associated real absolute frequency

$$
\omega_{0}^{c a}=\omega_{0}\left(X^{c a}\right) \quad \text { where } \quad \omega_{0, i}\left(X^{c a}\right)=0 .
$$

This condition is merely the linear frequency criterion put forward by Dee \& Langer (1983), according to which the front velocity is such that, in the co-moving frame, the 
medium is marginally absolutely unstable. The associated spatial structure consists of a stationary sharp front located at the transition station and separating an upstream decaying tail from a finite-amplitude downstream wavetrain. The front acts as a wave maker and imposes its frequency on the entire flow. In contrast with soft global modes, the wavenumber exhibits a sharp jump across the front. Similar nonlinear states with sharp Dee-Langer-type fronts were previously identified in amplitude evolution models of solar and stellar magnetic activity cycles by Meunier et al. (1997). The reader is referred to Soward (2001) for a review of related WKBJ asymptotic studies in the astrophysical context.

The transition scenarii towards fully nonlinear global modes have been analysed by Pier (1999) and Pier, Huerre \& Chomaz (2001) for the CGL evolution model in an infinite domain. The results of interest to the present investigation are as follows. The onset of steep global modes takes place via a saddle node bifurcation as soon as a point of local linear absolute instability appears within the medium. Since linear global modes in general become unstable only for a finite region of local linear absolute instability, the transition to a steep mode occurs while the medium is still globally linearly stable. Paradoxically, the onset of local linear absolute instability is seen to provide an accurate criterion for the bifurcation to fully nonlinear steep global modes while it is only a necessary condition for linear global instability. Local linear absolute instability in a sense prevails over global linear instability and dictates the nature of the observed finite-amplitude state.

The objective of the present study is to demonstrate that nonlinear self-sustained structures in real shear flows may be described in terms of steep global modes triggered by a stationary front located at the streamwise station of marginal absolute instability. Strong supporting evidence for this line of thought has recently been given by Delbende \& Chomaz (1998) in a direct numerical simulation of the nonlinear impulse response in a parallel wake. The nonlinear wavepacket is observed to be confined between the same leading and trailing edges as its linear counterpart. The sharp fronts delineating the saturated wavepacket then travel according to the linear Dee \& Langer (1983) velocity selection criterion.

In order to strictly enforce the weak streamwise non-uniformity condition underlying all the above notions, the basic flow must be carefully chosen. Bluff body wakes present a recirculation bubble near the obstacle which violates this assumption. To bypass this difficulty, we consider a 'synthetic' wake with no solid obstacle(!) and no reverse flow region, which still displays the essential features of wake flows, namely a region of local absolute instability. The basic ansatz is the same as in the numerical simulations of wakes 'without obstacles' performed by Triantafyllou \& Karniadakis (1990): vortex streets are produced by a wavemaker within the wake flow itself and the obstacle is only necessary in real laboratory experiments to generate the basic flow!

The outline of the study is as follows. The general formulation is presented in $\S 2$ together with the spatially developing basic wake flow derived as a solution of the Prandtl boundary layer equations. The ensuing local properties of linear instability waves are described in $\S 3$ in terms of a local linear dispersion relation at each downstream station. Corresponding local properties of fully nonlinear travelling waves are presented in $\S 4$ as solutions of a local nonlinear dispersion relation. These linear and nonlinear waves constitute the elementary building blocks of the globally synchronized structures in the upstream and downstream regions of the wake. Attention is then given in $\S 5$ to the missing link, namely the front structure supported by the wake profile at the marginally absolutely unstable station. Following the 
procedure proposed by Pier (1999) and Pier et al. (2001), the front is obtained as the limit state of the spatial response to time-harmonic forcing in a parallel wake, when the convective/absolute transition is reached. The self-sustained structures supported by the spatially developing basic flow are documented in $\S 6$, as computed by direct numerical simulation of the Navier-Stokes equations. Finally, $\S 7$ is concerned with the interpretation of these results in terms of the constitutive elements identified in the previous sections.

\section{General formulation and basic flow}

Two-dimensional incompressible flows are conveniently studied in terms of the streamfunction $\Psi(x, y, t)$ governed by the vorticity equation

$$
\left(\frac{\partial}{\partial t}+\frac{\partial \Psi}{\partial y} \frac{\partial}{\partial x}-\frac{\partial \Psi}{\partial x} \frac{\partial}{\partial y}\right) \Delta \Psi=\frac{1}{R e} \Delta^{2} \Psi .
$$

From the start non-dimensional variables based on the characteristic length and velocity scales $\ell^{\star}$ and $U^{\star}$ have been introduced (see below (2.5)). The Reynolds number is then defined as $R e=\ell^{\star} U^{\star} / v$, where $v$ is the kinematic viscosity. If $x$ and $y$ denote the streamwise and cross-stream coordinates, the corresponding $x$ and $y$ velocity components are obtained as $\partial \Psi / \partial y$ and $-\partial \Psi / \partial x$, and the vorticity as $-\Delta \Psi$.

The basic flow is steady and assumed to slowly develop in the streamwise direction. This is the case at large Reynolds numbers, where viscous spreading takes place on a slow streamwise scale. The order of magnitude of the weak streamwise non-uniformity is then effectively defined as

$$
\epsilon=\frac{1}{R e} \ll 1
$$

which is the only small parameter of the present investigation. Under this quasiparallel flow approximation, the basic flow streamfunction $\Psi_{b}$ only depends on $y$ and the slow streamwise coordinate

$$
X=\epsilon x,
$$

and it is expanded as

$$
\Psi_{b}(y ; X) \sim \Psi_{0}(y ; X)+\epsilon \Psi_{1}(y ; X)+\epsilon^{2} \Psi_{2}(y ; X)+\ldots .
$$

The leading-order basic flow approximation $\Psi_{0}$ is then readily shown to obey the Prandtl boundary layer equation

$$
\left(\frac{\partial \Psi_{0}}{\partial y} \frac{\partial}{\partial X}-\frac{\partial \Psi_{0}}{\partial X} \frac{\partial}{\partial y}\right) \frac{\partial \Psi_{0}}{\partial y}=-\frac{\mathrm{d} P}{\mathrm{~d} X}+\frac{\partial^{3} \Psi_{0}}{\partial y^{3}},
$$

where the given streamwise pressure gradient $\mathrm{d} P / \mathrm{d} X$ is the integration 'constant' arising in the integration of the leading-order vorticity equation. Corresponding basic velocity components are then $U_{0}(y ; X)=\partial \Psi_{0} / \partial y$ and $\epsilon V_{1}(y ; X)=-\epsilon \partial \Psi_{0} / \partial X$.

The basic flow is affected by viscous diffusion and by the externally applied pressure field $P(X)$ which allows its streamwise evolution to be precisely controlled. The parabolic boundary layer equation (2.4) is numerically integrated from $X=0$, with the $\operatorname{sech}^{2}$ inlet velocity profile

$$
U_{0}(y ; 0)=1-\frac{\Delta U}{\cosh ^{2}\left(y \sinh ^{-1} 1\right)} .
$$

Such a non-dimensional representation holds when the previously introduced velocity 


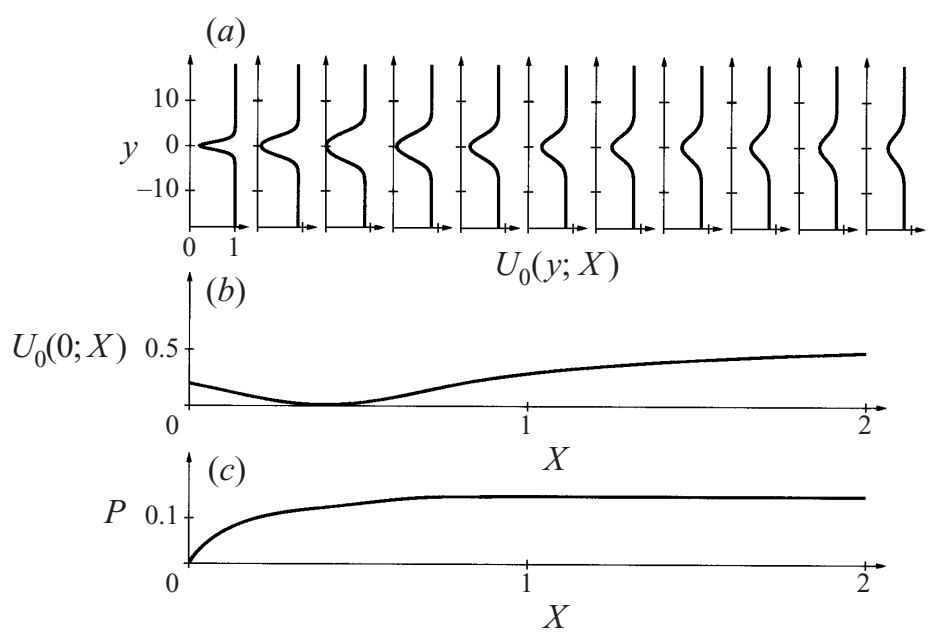

FIGURE 1. Streamwise development of the basic flow. (a) Velocity profiles $U_{0}(y ; X)$ at different streamwise $X$ locations. $(b)$ Evolution of centreline velocity $U_{0}(0 ; X)$. The smallest value $U_{0}(0 ; X)=0.01$ is reached at $X=0.4$. (c) Basic pressure field $P(X)$.

scale $U^{\star}$ is specified to be the streamwise basic flow inlet velocity at $y= \pm \infty$. The length scale $\ell^{\star}$ is chosen to be the half-width of the wake so that, in non-dimensional variables, $U_{0}(1 ; 0)=\frac{1}{2}[U(\infty ; 0)+U(0 ; 0)]$. The non-dimensional parameter $\Delta U$ measures the depth of the wake at the inlet and, in the present paper, we choose $\Delta U=0.8$.

In the upstream part of the flow, an adverse pressure gradient is carefully tailored in order to generate a central region of absolute instability, while avoiding reverse flow. The characteristics of the resulting basic flow are displayed in figure 1 . For the adverse pressure gradient sketched in figure $1(c)$, the centreline velocity (figure $1 b$ ) decreases from $1-\Delta U=0.2$ at the inlet to reach a minimum 0.01 at the streamwise station $X=0.4$. The relatively strong adverse pressure gradient close to the inlet slows down the basic flow while increasing the wake depth and rounding off the dip of the profile (figure 1a). Further downstream a uniform pressure is reached and thus the flow evolves solely under the action of viscosity: the wake depth decreases and slow diffusive spreading takes place.

Note that the Reynolds number in the boundary layer equation (2.4) has been scaled out and incorporated in the slow streamwise variable $X=\epsilon x$. As a result, the above basic flow applies to all large Reynolds numbers: changes in $R e$ are simply handled by a dilatation of the streamwise coordinate according to $x / X=R e$, the velocity profile shapes remaining unaltered.

The total streamfunction is then decomposed into steady basic and unsteady perturbation contributions according to

$$
\Psi(x, y, t)=\Psi_{b}(y ; X)+\psi(x, y, t) .
$$

Substitution of (2.6) with (2.3) into the governing equation (2.1), yields

$$
\begin{aligned}
\Delta \psi_{t} & +\Psi_{0, y} \Delta \psi_{x}-\Psi_{0, y y y} \psi_{x}+\left(\psi_{y} \partial_{x}-\psi_{x} \partial_{y}\right) \Delta \psi \\
& +\epsilon\left[\Psi_{1, y} \Delta \psi_{x}-\Psi_{0, X} \Delta \psi_{y}-\Psi_{1, y y y} \psi_{x}+\Psi_{0, X y y} \psi_{y}-\Delta^{2} \psi\right]=\mathcal{O}\left(\epsilon^{2}\right),
\end{aligned}
$$

where the subscripts $t, x, y$ and $X$ denote differentiation with respect to these variables. 
The slow streamwise variations of the basic flow call for an analysis of the perturbation field in terms of both linear and nonlinear WKBJ approximations (Bender \& Orszag 1978). The properties of local linear and nonlinear wavetrain solutions sustained by the basic flow are derived in the following two sections.

\section{Local linear instability waves}

Following e.g. Crighton \& Gaster (1976) and Monkewitz et al. (1993), in the linear approximation $\psi$ is sought in the form of a slowly varying wavetrain of frequency $\omega$ :

$$
\psi(x, y, t)=\psi_{\star}(y ; X) \exp \left(\frac{\mathrm{i}}{\epsilon} \int^{X} k(u) \mathrm{d} u-\mathrm{i} \omega t\right)+\text { c.c. },
$$

where $k(X)$ denotes the local complex wavenumber and

$$
\psi_{\star}(y ; X) \sim \psi_{0}(y ; X)+\epsilon \psi_{1}(y ; X)+\ldots
$$

accounts for the local cross-stream structure. Substitution of expressions (3.1), (3.2) into the linearized version of (2.7) shows that the leading-order approximation $\psi_{0}$ is governed by the local Rayleigh equation

$$
\left(k U_{0}(y ; X)-\omega\right)\left(\frac{\partial^{2} \psi_{0}}{\partial y^{2}}-k^{2} \psi_{0}\right)-k U_{0, y y}(y ; X) \psi_{0}=0 .
$$

Linear eigenmodes $\Psi^{\ell}(y ; k, X)$ that are solutions of (3.3) and exponentially decaying at $y= \pm \infty$ are obtained when the complex frequency $\omega$ and complex wavenumber $k$ are bound by the local linear dispersion relation

$$
\omega=\Omega^{\ell}(k, X),
$$

at each station $X$. For a given wavenumber and a given wake velocity profile there exists a set of eigenfunctions to the Rayleigh equation. We only consider the sinuous mode ( $\psi$ even) since it is the most amplified (Drazin \& Reid 1981). In what follows, the functions $\Psi^{\ell}$ and $\Omega^{\ell}$ always refer to this particular eigenfunction and eigenvalue respectively. For definiteness, the linear $\Psi^{\ell}$ eigenfunctions are normalized by $\Psi^{\ell}(0 ; k, X)=1$.

The Rayleigh equation (3.3) has been solved via a Chebyshev spectral method (Canuto et al. 1988). The Chebyshev collocation points $-1 \leqslant \xi_{i}=-\cos \left[i \pi /\left(n_{y}+1\right)\right] \leqslant+1$ for $i=0, \ldots, n_{y}+1$ are mapped onto $-\infty \leqslant y_{i} \leqslant+\infty$ through the transformation

$$
\frac{\sqrt{2}}{l_{y}} y=\frac{\xi}{1-\xi^{2}} \text {. }
$$

The parameter $l_{y}$ dictates the distribution of collocation points on the $y$-axis: half are located in the interval $-l_{y}<y<l_{y}$ and are approximately equispaced; the remaining points extend towards $y= \pm \infty$ and their density decreases algebraically. For a given $k$, the differential Rayleigh equation is thus recast into a linear eigenvalue problem: the frequency $\omega$ and cross-stream distribution $\Psi^{\ell}$ are obtained as eigenvalues and eigenfunctions respectively. As many eigenfunctions as collocation points are obtained. The relevant sinuous eigenfrequency is then identified by inspection of its eigenfunction. The complete linear dispersion relation $\Omega^{\ell}(k, X)$ is generated by continuation in $k$ and $X$. Note that, since the eigenfunctions $\Psi^{\ell}$ exponentially decay towards $y= \pm \infty$ as $\mathrm{e}^{-\left|k_{r} y\right|}$, the transformation (3.5) is appropriate for this problem. In general $n_{y}=50$ collocation points with $l_{y}=5$ are found to be sufficient to accurately 


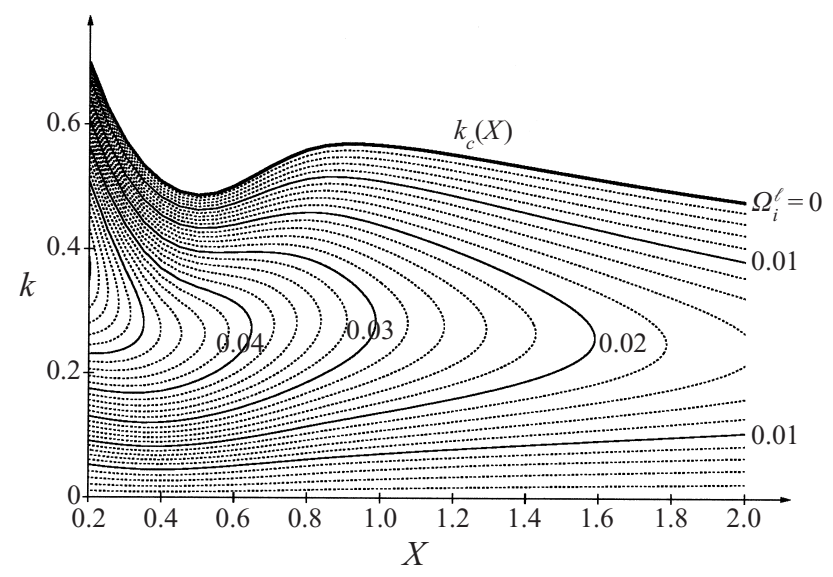

FIGURE 2. Isocontours of sinuous mode temporal growth rates $\Omega_{i}^{\ell}$ in the $(X, k)$-plane in the interval $0.2<X<2.0$ of figure 1 . Solid contours are separated by $\delta \Omega_{i}^{\ell}=0.01$, dashed contours by $\delta \Omega_{i}^{\ell}=0.002$. Larger growth rates occur in the upstream region whereas instability weakens further donwnstream. The unstable domain is bounded by the neutral curve $k_{c}(X)$ (thick line).

resolve the eigenfunction and precisely obtain the frequency; however for small values of $k_{r}$ a larger $n_{y}$ is necessary. As a validation, the mode shape is required not to vary when doubling the number of collocation points.

The dispersion relation (3.4) yields the local wavenumber $k^{\ell}(X, \omega)$ as a function of the global frequency. Thus at leading order in the WKBJ expansion

$$
\psi(x, y, t) \sim A_{0}(X) \Psi^{\ell}\left(y ; k^{\ell}(X, \omega), X\right) \exp \left(\frac{\mathrm{i}}{\epsilon} \int^{X} k^{\ell}(u, \omega) \mathrm{d} u-\mathrm{i} \omega t\right)+\text { c.c. },
$$

where the slowly varying complex amplitude function $A_{0}(X)$ could be determined at next order by a secularity condition.

Local linear instability is characterized by the temporal growth of real wavenumbers. Figure 2 illustrates the streamwise evolution of sinuous temporal growth rates $\Omega_{i}^{\ell}(k, X) \equiv \operatorname{Im} \Omega^{\ell}(k, X)$ in the $(X, k)$-plane for the basic flow of figure 1. All wake profiles exhibit long-wavelength instability and the wake flow remains linearly unstable far downstream. However, the maximum temporal growth $\operatorname{rate} \operatorname{Max}\left\{\Omega_{i}^{\ell}(k, X), k\right.$ real $\}$ decreases with $X$. The unstable domain in the $(X, k)$-plane is defined by the condition $\Omega_{i}^{\ell}(k, X)>0$. Its boundary yields the neutral curve $k_{c}(X)$ (thick line in figure 2) associated through (3.4) with a real frequency. Linear growth of unstable waves is limited by nonlinear saturation effects: the corresponding nonlinear wavetrains are then obtained as discussed in the next section.

The local complex absolute frequency $\omega_{0}(X)$ and absolute wavenumber $k_{0}(X)$ are defined via dispersion relation (3.4) as

$$
\omega_{0}(X)=\Omega^{\ell}\left(k_{0}(X), X\right) \quad \text { with } \quad \frac{\partial \Omega^{\ell}}{\partial k}\left(k_{0}(X), X\right)=0 .
$$

The locus of $\omega_{0}(X)$ pertaining to the basic wake flow is represented in the complex $\omega$-plane in figure $3(a)$ and the corresponding streamwise variation of absolute growth rate $\omega_{0, i}(X)$ is sketched in figure $3(b)$. According to these sketches, the properties of the flow under consideration present the following desirable features. The upstream region extending from the inlet at $X=0$ to $X^{c a}=0.24$ is convectively unstable (CU). The adverse pressure gradient prevailing in this domain induces an increase of $\omega_{0, i}$ 


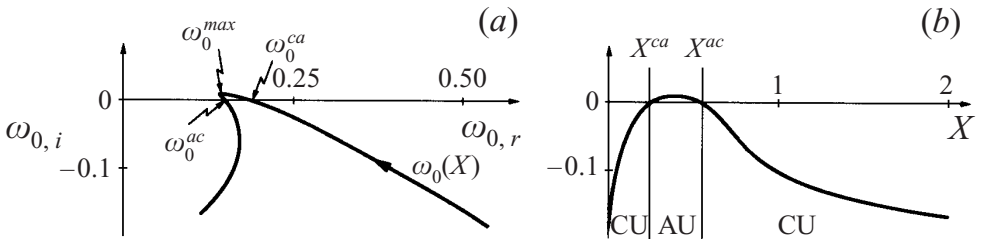

FIGURE 3. (a) Locus of local absolute frequency $\omega_{0}(X)$ in the complex frequency plane.

(b) Local absolute growth rates $\omega_{0, i}(X)$ as a function of streamwise station.

which leads to absolute instability at $X^{c a}$. Beyond this station of marginal absolute instability, the flow displays a central absolutely unstable (AU) region extending over the interval $X^{c a}<X<X^{a c}$, with $X^{a c}=0.55$. In the absence of further adverse pressure gradient, the flow then returns to convective instability far downstream $X>X^{a c}$

According to Pier et al. (1998), the boundaries of the AU region are of particular interest. These specific locations are defined by real absolute frequencies, $\omega_{0}^{c a} \equiv \omega_{0}\left(X^{c a}\right)=0.190$ and $\omega_{0}^{a c} \equiv \omega_{0}\left(X^{a c}\right)=0.150$ respectively. Note that $\omega_{0}^{c a}>\omega_{0}^{a c}$, a feature which has been observed for all wake flows of the present study. Although not essential to the observation of steep global modes, this inequality was taken as a working assumption in Pier et al. (2001). The largest absolute growth rate occurs at $X^{\max }=0.38$ where $\omega_{0}^{\max } \equiv \omega_{0}\left(X^{\max }\right)=0.144+0.010 \mathrm{i}$.

Following Chomaz et al. (1991) and Monkewitz et al. (1993), we may analyse the linear global instability of the present flow by applying the saddle point criterion (1.1). The analytic continuation of $\omega_{0}(X)$ in the complex $X$-plane yields a saddle point at $X_{s}=0.43+0.03 i$, associated with a linear global mode of frequency $\omega_{s}=0.143+0.008 \mathrm{i}$. Since $\omega_{s, i}>0$, the wake flow is linearly globally unstable.

\section{Local fully nonlinear travelling waves}

In regions where perturbations reach finite amplitude levels, the flow is governed by the complete nonlinear equation (2.7). The experiments of Provansal et al. (1987) as well as the numerical simulations of Hammond \& Redekopp (1997) reveal that nonlinear structures in wakes are propagating in the streamwise direction: they are locally periodic and their $(x, t)$-dependence occurs solely via a phase function with only slow streamwise deformations. Guided by these observations and following the WKBJ formalism, we seek nonlinear slowly varying wavetrains to equation (2.7) of the form

$$
\psi(x, y, t)=\psi_{\star}(\theta, y ; X) \quad \text { with } \quad \theta=\frac{1}{\epsilon} \int^{X} k(u) \mathrm{d} u-\omega t,
$$

where $\psi_{\star}$ is a $2 \pi$-periodic function of $\theta$, and $k(X)$ is the real local wavenumber. Following the nonlinear WKBJ approximation scheme of Pier \& Huerre (1996) and Pier et al. (2001), the nonlinear wavetrain is again expanded as

$$
\psi_{\star}(\theta, y ; X) \sim \psi_{0}(\theta, y ; X)+\epsilon \psi_{1}(\theta, y ; X)+\ldots .
$$

At leading order, one obtains

$$
\begin{aligned}
\left(k U_{0}(y ; X)-\omega\right) & \frac{\partial}{\partial \theta}\left(\frac{\partial^{2} \psi_{0}}{\partial y^{2}}+k^{2} \frac{\partial^{2} \psi_{0}}{\partial \theta^{2}}\right)-k U_{0, y y}(y ; X) \frac{\partial \psi_{0}}{\partial \theta} \\
+ & k\left(\frac{\partial \psi_{0}}{\partial y} \frac{\partial}{\partial \theta}-\frac{\partial \psi_{0}}{\partial \theta} \frac{\partial}{\partial y}\right)\left(\frac{\partial^{2} \psi_{0}}{\partial y^{2}}+k^{2} \frac{\partial^{2} \psi_{0}}{\partial \theta^{2}}\right)=0 .
\end{aligned}
$$


As shown below, this partial differential equation in $\theta$ and $y$ admits periodic solutions $\Psi^{n \ell}(\theta, y ; k, X)$ when the real frequency $\omega$ and the real wavenumber $k$ are related by the local nonlinear dispersion relation

$$
\omega=\Omega^{n \ell}(k, X),
$$

at each station $X$. The dispersion relation (4.4) yields the local nonlinear wavenumber $k^{n \ell}(X, \omega)$ as a function of the global frequency. Thus at leading order in the nonlinear WKBJ expansion

$$
\psi(x, y, t) \sim \Psi^{n \ell}\left(\frac{1}{\epsilon} \int^{X} k^{n \ell}(u, \omega) \mathrm{d} u-\omega t+\theta_{0}(X), y ; k^{n \ell}(X, \omega), X\right),
$$

where the slowly varying phase function $\theta_{0}(X)$ could be determined at next order by a solvability condition.

Due to the separation of scales, the phase function $\theta$ governs the fast oscillations on a typical instability length scale, whereas $X$ accounts for the slow development of the basic velocity profile and is not involved in spatial differentiation. Hence local properties at a given streamwise station $X$ are recovered by freezing the $X$-dependence of the velocity profile and studying the corresponding strictly parallel flow. Thus the term 'local' always refers to properties of strictly parallel flows obtained by extending towards $x= \pm \infty$ the velocity profile prevailing at a specific downstream station $X$.

The local nonlinear travelling waves $\Psi^{n \ell}(\theta, y ; k, X)$ are now shown to arise naturally from a purely temporal analysis of the strictly parallel flow $U_{0}(y ; X)$ obtained by freezing $X$. To this extent, the slow coordinate $X$ is regarded as an external control parameter, frozen at a constant value although $x$ varies on the entire real axis. The initial value problem of interest is the study of the development of a spatially periodic perturbation of given real wavenumber $k$. The initial evolution of such a small-amplitude perturbation is dictated by the linear temporal growth rate $\Omega_{i}^{\ell}(k, X)$. Whenever $\Omega_{i}^{\ell}>0$, its amplitude exponentially grows until nonlinearity sets in. Stabilizing nonlinearities lead to saturation at finite amplitude and, at large time, a fully nonlinear solution is obtained with streamwise periodicity imposed by the initial wavenumber $k$. In the absence of secondary instabilities, a propagating nonlinear wave solution is reached, and its frequency yields the nonlinear dispersion relation (4.4). The same thought experiment may be carried out at each streamwise station $X$. It should be emphasized that, without viscous dissipation, the above procedure does not single out a unique attracting wavetrain solution onto which the system converges for large time. In order to circumvent this difficulty, we have chosen to regard the pertinent finite-amplitude states as long-time saturated solutions of the full viscous vorticity equation

$$
\left(\frac{\partial}{\partial t}+U_{0}(y) \frac{\partial}{\partial x}\right) \Delta \psi_{0}-U_{0, y y}(y) \frac{\partial \psi_{0}}{\partial x}+\left(\frac{\partial \psi_{0}}{\partial y} \frac{\partial}{\partial x}-\frac{\partial \psi_{0}}{\partial x} \frac{\partial}{\partial y}\right) \Delta \psi_{0}=\frac{1}{R e} \Delta^{2} \psi_{0},
$$

for the perturbation stream function $\psi_{0}$. In the above expression, the basic flow is strictly parallel and its parametric dependence on $X$ has been omitted. Note that the viscous term $\Delta^{2} \psi_{0} / R e$ would only appear at higher order if the WKBJ asymptotic scheme were strictly applied. The use of a large but finite $R e$ is brought in to select a unique periodic nonlinear wavetrain. The precise value of $R e$ will later be proven unimportant.

One should also note that such a solution can only be preserved at large time if the basic wake shear is maintained steady and parallel. In the calculation, this is achieved by separating basic and perturbation fields. In effect, as in the direct 
numerical simulations of shear flows, e.g. Ehrenstein \& Koch (1995), it is implicitly assumed that a body force is present to counteract viscous diffusion of the basic flow. Note that the extra terms introduced in the equations for the basic flow and perturbations are higher-order corrections: they are introduced for computational convenience, and in the limit $\epsilon=1 / R e \rightarrow 0$ the original equation is recovered.

The temporal evolution of an initial spatially periodic perturbation $\psi_{0}(x, y, t)$ of real wavenumber $k$ is treated by solving (4.6) on a streamwise periodic interval of given wavelength $2 \pi / k$. The temporal linear sinuous eigenmode of real wavenumber $k$ is chosen as initial perturbation, and, in the linear régime, it evolves according to $\Omega^{\ell}(k, X)$. Whenever $\Omega_{i}^{\ell}(k, X)>0$, it is amplified, and nonlinear terms come into play to promote higher harmonics as well as a non-fluctuating mean flow correction. The long-time finite-amplitude state is precisely the nonlinear travelling wave $\psi_{0}(x, y, t)=\Psi^{n \ell}(k x-\omega t, y)$ solution of frequency $\omega$ of the nonlinear dispersion relation (4.4) for the basic flow at the station $X$ under consideration. In order to complete the specification of the temporal evolution problem, we assume that the flow rate is unaltered by the presence of the finite-amplitude perturbations.

An example of a nonlinear travelling wave state of wavenumber $k=0.30$ is illustrated in figure 4 for the basic velocity field (thick line in figure $4 a$ ) at $X=1.0$. This wavenumber is linearly amplified according to $\Omega^{\ell}=0.183+0.029 \mathrm{i}$. Beyond a transient régime, a periodic state is reached, at the numerically determined nonlinear frequency $\Omega^{n \ell}(k, X)=0.205$. In figure $4(a)$, the basic velocity profile $U_{0}(y)$ (thick line) is compared with the nonlinear mean flow correction $u_{(0)}(y)$ (thin line) and the total mean flow $U_{0}+u_{(0)}$ (dashed line). The non-fluctuating component of the perturbation field is seen to essentially reduce the depth of the wake and to increase its width. Contour levels of the perturbation vorticity field $-\Delta \psi_{0}$ are displayed in figure $4(b)$. The periodic spatial structure is seen to consist of a double row of counter-rotating vortices as one would expect in a Kármán vortex street. The main vortex street is surrounded by two vortex rows of smaller amplitude and opposite sign. The whole nonlinear structure propagates in the downstream direction at the celerity $\Omega^{n \ell} / k=0.68$, slower than the far-field basic velocity $U_{0}( \pm \infty)=0.84$. Since the basic flow is maintained, such a periodic state persists at large time and the nonlinear frequency is well defined. Note however that the mean flow correction $u_{(0)}(y)$ (thin line in figure $4 a$ ) is observed to slowly diffuse in the $y$-direction over a slow viscous time scale.

Such nonlinear states naturally only exist in the unstable domain of the $(X, k)$-plane, defined by a positive linear growth rate (figure 2). Isocontours of $\Omega^{n \ell}$ in this region are represented in figure $5(a)$ and they should be compared to the corresponding isocontours of $\Omega_{r}^{\ell}$ displayed in figure $5(b)$. At the neutral stability boundary (thick curves in figure 5), the linear temporal growth rate vanishes and the finite-amplitude wavetrains smoothly turn into the linear neutral eigenmodes. As a result, on the neutral curve

$$
\Omega^{n \ell}(k, X)=\Omega_{r}^{\ell}(k, X) \quad \text { and } \quad \Omega_{i}^{\ell}(k, X)=0 .
$$

Note that linear and nonlinear frequencies identically vanish for $k=0$.

A careful comparison of figures $5(a)$ and $5(b)$ reveals that the nonlinear frequencies are always larger than the linear ones. Thus nonlinear interactions tend to increase the celerity $\omega / k$ of the wavetrains. It should be emphasized that the nonlinear isofrequency curves in the $(X, k)$-plane displayed in figure $5(a)$ precisely coincide with the nonlinear spatial branches $k^{n \ell}(X, \omega)$ at the corresponding frequency, as introduced in the nonlinear wavetrain (4.5). 
(a)
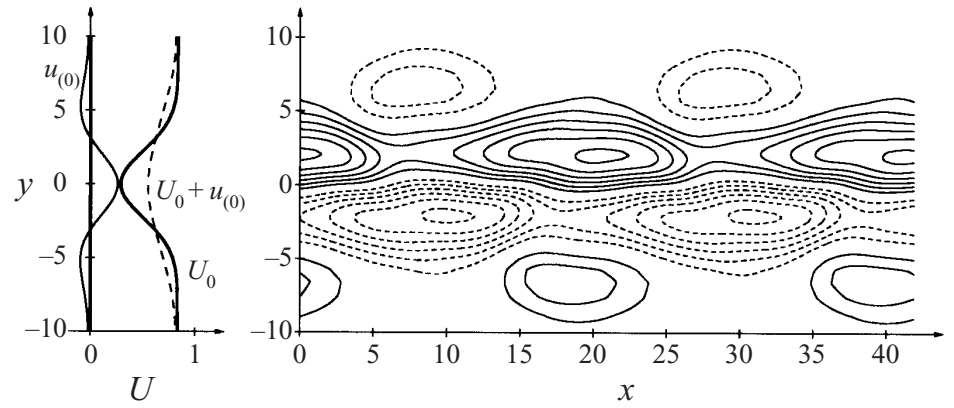

FiguRE 4. Local nonlinear travelling wave of wavenumber $k=0.30$ at the downstream station $X=1.0$ with $R e=100$ at time $t=6000$. (a) Basic velocity profile $U_{0}(y)$ (thick line), meanflow correction $u_{(0)}(y)$ (thin line) and total mean flow $U_{0}(y)+u_{(0)}(y)$ (dashed line). (b) Isolines of the perturbation vorticity field over two wavelengths. Positive isolines $0.025,0.050, \ldots, 0.150$ and negative isolines $-0.025,-0.050, \ldots,-0.150$ are represented by solid and dashed curves respectively.
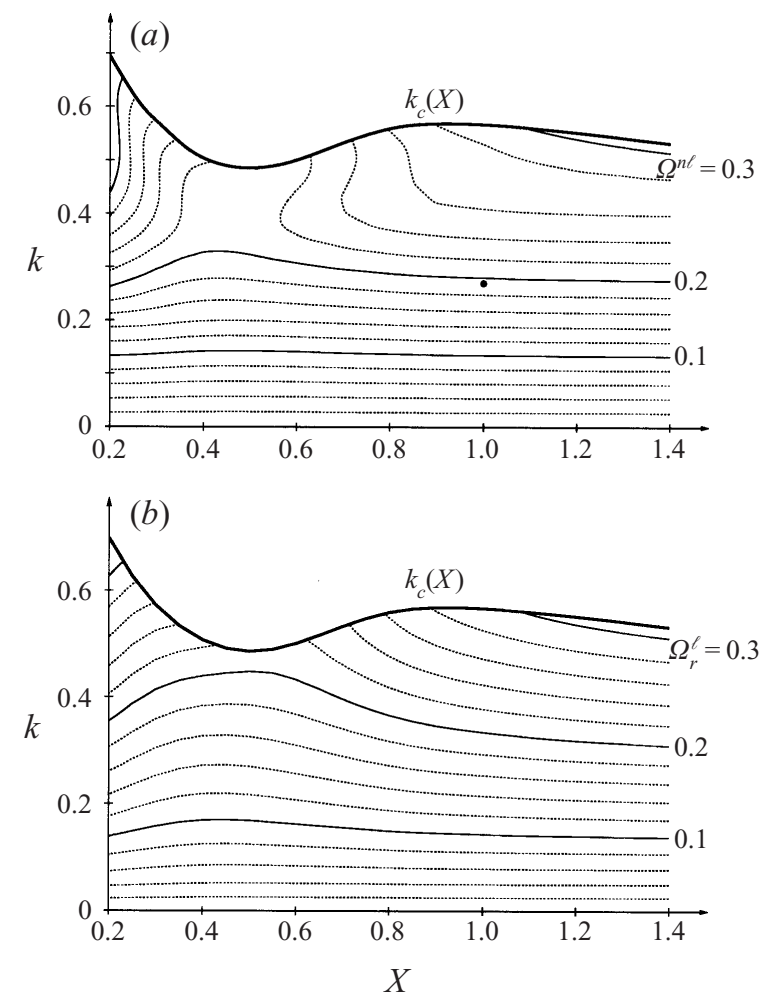

FIGURE 5. Isofrequency contours in the linearly unstable domain of the $(X, k)$-plane. Solid contours are separated by $\delta \Omega=0.1$, dashed contours by $\delta \Omega=0.02$. (a) Isocontours of $\Omega^{n \ell}$. (b) Isocontours of $\Omega_{r}^{\ell}$.

The numerical technique used to solve (4.6) is based on the spectral method of Ehrenstein \& Koch (1989) suitably adapted to wake flows. The streamfunction is decomposed into harmonic components as

$$
\psi_{0}(x, y, t)=\sum_{-\infty<n<\infty} \phi_{(n)}(y, t) \mathrm{e}^{\mathrm{i} n k x},
$$


with $\phi_{(-n)}(y, t)=\phi_{(n)}^{\star}(y, t)$, where $\star$ denotes the complex conjugate. Substitution of (4.7) into the governing equation (4.6) and identification of corresponding exponentials leads to the modal equations

$$
\left[\partial_{t} \mathrm{D}_{n}+\mathrm{i} n k\left[U_{0}(y) \mathrm{D}_{n}-U_{0, y y}(y)\right]-\frac{1}{R e} \mathrm{D}_{n}^{2}\right] \phi_{(n)}+\mathrm{i} k \sum_{-\infty<m<\infty} N_{n-m, m}=0,
$$

where $\mathrm{D}_{n}=\partial_{y y}-(n k)^{2}$ and

$$
N_{n, m}=m\left(\partial_{y} \phi_{(n)}\right)\left(\mathrm{D}_{m} \phi_{(m)}\right)-n \phi_{(n)}\left(\mathrm{D}_{m} \partial_{y} \phi_{(m)}\right) .
$$

Assuming that the nonlinear perturbation does not change the pressure gradient at $y= \pm \infty$ and leaves the flow rate unaltered, equation (4.8) may be integrated twice for $n=0$ to obtain the mean flow distortion equation

$$
\frac{\partial \phi_{(0)}}{\partial t}-\frac{1}{R e} \frac{\partial^{2} \phi_{(0)}}{\partial y^{2}}=2 k \operatorname{Im} \sum_{m>0} m \phi_{(m)}^{\star} \frac{\partial \phi_{(m)}}{\partial y} .
$$

The unknown functions $\phi_{(n)}(y, t)$ all vanish at $y= \pm \infty$, and the mean flow correction reads $u_{(0)}=\partial \phi_{(0)} / \partial y$.

The set of modal equations (4.8), (4.10) is truncated at a finite number of harmonics $|n| \leqslant n_{h}$ and again a Chebyshev collocation method with $n_{y}$ collocation points is implemented in the $y$-direction together with the mapping (3.5). Thus the governing equation (4.6) has been recast as a system of $n_{y}\left(2 n_{h}+1\right)$ real algebraic ordinary differential equations of the first order in time. These are solved via a fourth-order Runge-Kutta scheme of time step $\delta t$. In the particular example of figure 4 at $R e=100$ and $t=6000, n_{h}=5, n_{y}=71, l_{y}=5$ and $\delta t=0.05$. It has been checked that higher resolutions leave the results unchanged.

The temporal evolution of the wavenumber spectrum is illustrated in figure 6 . The kinetic energy $E_{n}(t)$ of each harmonic is sketched as a function of time on both logarithmic and linear scales. If $u_{(n)}=\partial_{y} \psi_{(n)} \mathrm{e}^{\mathrm{i} n k x}+$ c.c. and $v_{(n)}=-\mathrm{i} n k \psi_{(n)} \mathrm{e}^{\mathrm{i} n k x}+$ c.c. denote the velocity field associated with the $n$th harmonic $(n>0)$, its energy is defined as

$$
E_{n}(t)=\int_{-\infty}^{+\infty} \int_{0}^{2 \pi / k} \frac{1}{2}\left(u_{n}^{2}+v_{n}^{2}\right) \mathrm{d} x \mathrm{~d} y .
$$

During the linear growth phase $(t<800$, figure $6 a)$, all harmonics are exponentially amplified. The measured slope of the fundamental $E_{1}$ (thick line) in the semi-log plot is 0.0255 , and precisely coincides with its theoretical value given by $2 \Omega_{i}^{\ell} / \ln 10$. Higher harmonics $E_{n}(t)(n>1$, dashed lines) are naturally slaved to the fundamental and grow as $\left[E_{1}(t)\right]^{n}$ whereas the mean flow correction $E_{0}(t)$ (thin solid line) scales as $\left[E_{1}(t)\right]^{2}$.

Beyond the nonlinear transient régime $(800<t<2000)$, the system relaxes towards a quasi-stationary travelling wave state. The duration of the transients depends on the dissipation time scale as dictated by the magnitude of the selected Reynolds number. The choice $R e=100$ appears to be a good compromise between reasonably long transients and inviscid-like dynamics. Although the motion is fully nonlinear, only a few harmonic components partake in the dynamics. Even for the most unstable wavenumber, only three harmonics are necessary to accurately capture the saturated wave state.

The final nonlinear propagating wave and its frequency hardly depend on the particular value of the Reynolds number, as illustrated in figure 7. For $R e>50$, the frequency changes by less than 0.0025 . It is only for $R e<20$ that drastic changes 

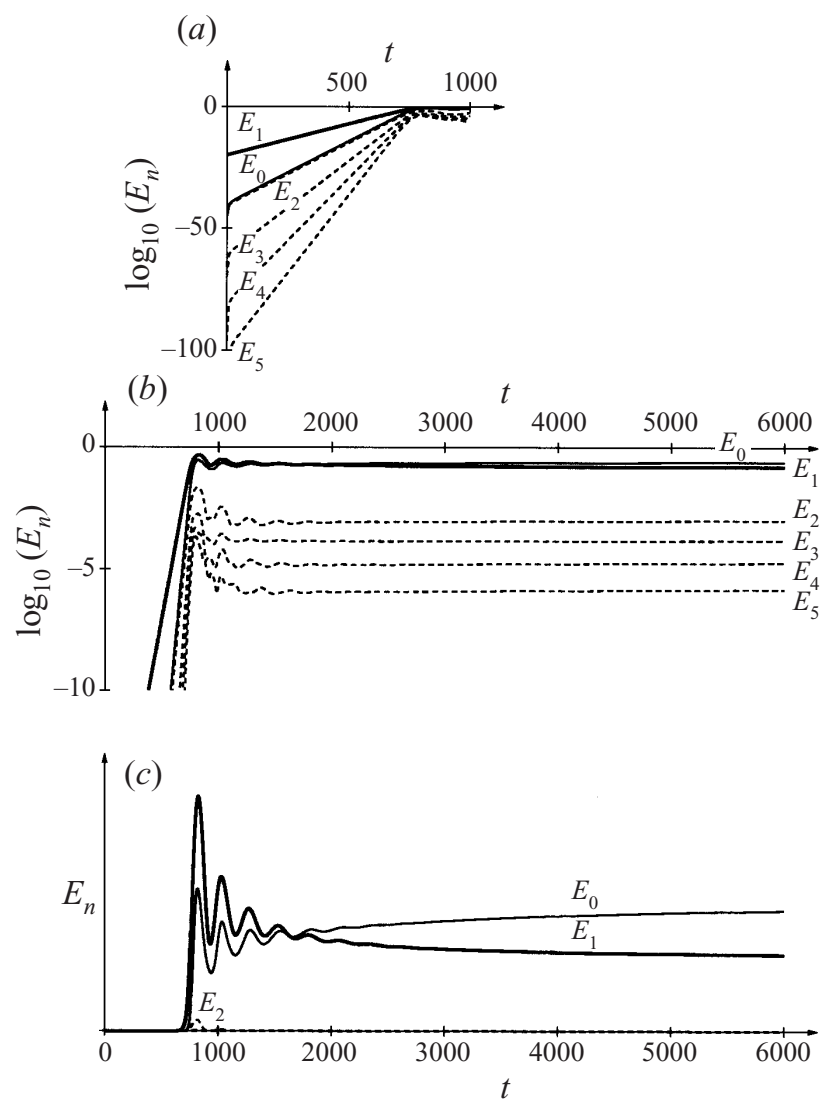

FiguRE 6. Temporal evolution of the energy contained in each harmonic component (nonlinear travelling wave of figure 4) for $0<t<6000$. (a) Semi-log plot of the initial linear growth phase $(t<800)$. The fundamental $E_{1}$ (thick solid line) grows acccording to exp $2 \Omega_{i}^{\ell} t$, higher harmonics $E_{n}\left(n>1\right.$, dashed lines) are slaved as the $n$th power of $E_{1}$, the mean flow correction $E_{0}$ as $E_{1}^{2}$. (b,c) Semi-log and linear plots of the long time evolution display a nonlinear transient régime $(800<t<2000)$ with damped oscillations towards a quasi-stationary state. Note that the harmonics $(n>2)$ reach much lower saturation levels.

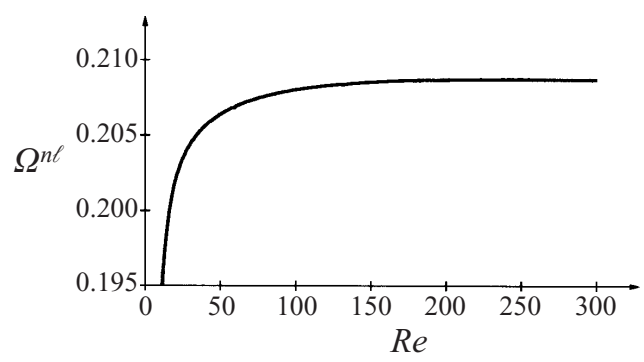

FIGURE 7. Variation of the nonlinear frequency $\Omega^{n \ell}$ associated with $k=0.30$ and $X=1.0$ as a function of the Reynolds number Re. Note the magnified vertical scale.

in the frequency occur! This insensitivity to Reynolds number fully justifies the approach adopted in the present study. The addition of the viscous term in (4.6) does not significantly affect the properties of the nonlinear wavetrain. In the present calculation, the value $R e=100$ has been deemed sufficient. 


\section{Nonlinear spatial response and fronts in parallel wakes}

At each downstream location we have now at our disposal a complete family of linear as well as nonlinear local wavetrains which constitute the elementary building blocks of a globally synchronized flow. Whereas the upstream region extending to the inlet is expected to sustain small perturbation levels, correctly handled within a linear approximation, the downstream wake should be made up of fully nonlinear travelling wavetrains. In the framework of one-dimensional evolution models, it has been demonstrated (Pier 1999) that, for steep global modes, a stationary front constitutes the missing link converting the upstream linear waves into the downstream nonlinear waves at a station of marginal local absolute instability. In this section it is shown that an analogous front structure exists in wake flows. According to Dee \& Langer (1983) and van Saarloos $(1987,1988)$, a propagating front moves towards its decaying edge if the basic state is $\mathrm{AU}$, towards its finite-amplitude edge if the basic state is $\mathrm{CU}$, and a stationary front is obtained when the medium is exactly at the CU/AU transition.

Here a slightly different point of view is adopted: instead of studying front propagation, we focus on the spatial response of a CU profile to a localized time-harmonic forcing and argue that the stationary front is recovered at the CU/AU transition. This approach is motivated by the difficulty in directly computing the propagation of a front connecting a fully nonlinear wavetrain to an unperturbed wake profile: in the co-moving frame the flow is marginally AU and thus very sensitive to various uncontrolled perturbations. In contrast, the signalling problem in a CU flow is not affected since the response to noise is swept away, and the stationary régime is easily identified.

Consider again a strictly parallel wake profile at a given streamwise station $X$. The forced perturbation vorticity and streamfunction fields $\varpi$ and $\psi$ are then governed by

$$
\begin{aligned}
\left(\frac{\partial}{\partial t}+U_{0}(y) \frac{\partial}{\partial x}\right) \varpi+U_{0, y y}(y) \frac{\partial \psi}{\partial x}+\left(\frac{\partial \psi}{\partial y} \frac{\partial}{\partial x}-\frac{\partial \psi}{\partial x} \frac{\partial}{\partial y}\right) \varpi & =\frac{\Delta \varpi}{R e}+S(x, y, t), \\
\Delta \psi & =-\varpi,
\end{aligned}
$$

where the temporally harmonic forcing function is taken to be of the form

$$
S(x, y, t)=H(t) A_{f} \exp \left(-\frac{x^{2}+\left(y-y_{f}\right)^{2}}{\ell_{f}^{2}}\right) \cos \omega_{f} t,
$$

with $H(t)$ denoting the Heaviside unit step function, and $\omega_{f}$ and $A_{f}$ the forcing frequency and amplitude. The forcing is applied in a region of size $\ell_{f}$ centred at $\left(0, y_{f}\right)$. Switching on the forcing at $t=0$ produces a transient wavepacket together with the steady-state response at the forcing frequency. Whenever the basic flow is $\mathrm{CU}$, transients move away from the source and a steady-state signal is left at the forcing frequency. When the medium is AU, switch-on transients overwhelm the response at the forcing frequency and the signalling problem is ill-posed. Hence only the spatial problem for $\mathrm{CU}$ velocity profiles is considered in the following discussion.

The linear signalling problem in shear flows has been investigated by Huerre \& Monkewitz (1985). Here, the linear dispersion relation (3.4) gives rise to two linear spatial branches $k^{\ell+}(X, \omega)$ and $k^{\ell-}(X, \omega)$. The response streamfunction then reads

$$
\psi(x, y, t) \propto A_{f} \frac{\Psi^{\ell}\left(y ; k^{\ell \pm}\left(X, \omega_{f}\right), X\right)}{\left(\partial \Omega^{\ell} / \partial k\right)\left(k^{\ell \pm}\left(X, \omega_{f}\right), X\right)} \operatorname{expi}\left[k^{\ell \pm}\left(X, \omega_{f}\right) x-\omega_{f} t\right]+\text { c.c. }
$$

where labels $(+)$ and $(-)$ pertain to the downstream $(x>0)$ and upstream $(x<0)$ 

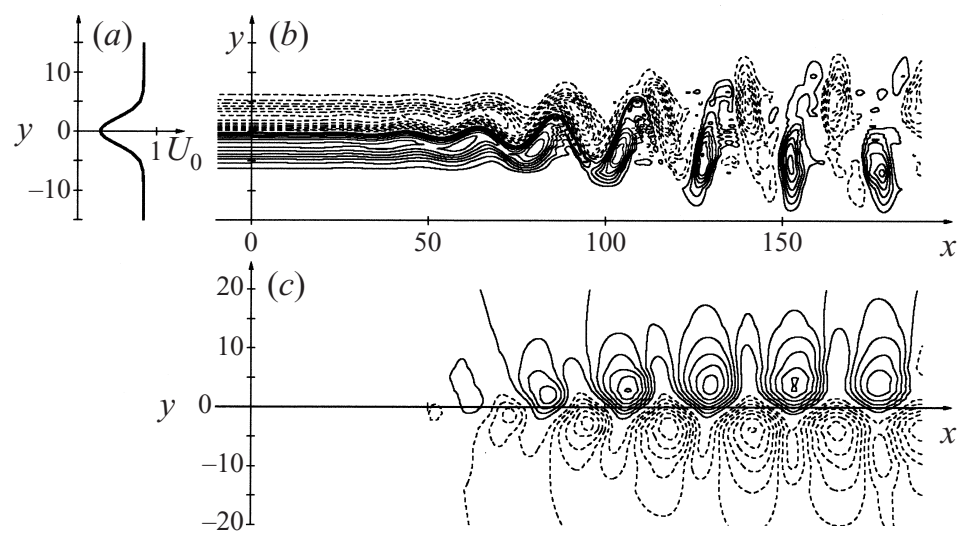

FiguRE 8. Spatial response of a CU parallel wake to harmonic forcing of frequency $\omega_{f}=0.19$, amplitude $A_{f}=10^{-3}$ and width $\ell_{f}=1$ applied at the origin and given by (5.2). $R e=100$. (a) Basic velocity profile at $X=1$. (b) Total vorticity levels at $t=500$. Positive contours $0.02,0.04, \ldots$, 0.14 and negative contours $-0.02,-0.04, \ldots,-0.14$ are represented by solid and dashed lines respectively. (c) Corresponding perturbation streamlines. Solid (dashed) isocontours pertain to the levels $0.2,0.4, \ldots, 1.4(-0.2,-0.4, \ldots,-1.4)$. Close to the forcing location the downstream response grows exponentially to reach a finite amplitude around $x=70$. Further downstream, a nonlinear travelling wave is generated. The entire streamwise extent $-10<x<190$ of the computational domain is shown.

response respectively. Note again that for convenience the viscous dissipation term $\Delta \varpi / R e$ has been added to $(5.1 a)$. Thus the linear dispersion relation $\Omega^{\ell}$ and corresponding eigenfunctions $\Psi^{\ell}$ are derived from the Orr-Sommerfeld equation rather than the Rayleigh equation (3.3), the streamwise decay or growth rates being dictated by $k_{i}^{\ell \pm} \equiv \operatorname{Im} k^{\ell \pm}$. An extensive survey of all the $\mathrm{CU}$ velocity profiles encountered in the present study reveals that the linear $k^{\ell-}(X, \omega)$ branches always remain in the $k_{i}<0$ half-plane and thus never give rise to upstream amplification. Only downstream spatial branches $k^{\ell+}$ grow in a certain frequency range.

Whenever a linear spatial branch is amplified, nonlinear terms have to be taken into account at some distance from the source, however small the forcing amplitude. As the response reaches finite amplitude, nonlinear saturation prevents further amplification and leads to a nonlinear travelling wave at the excitation frequency for some real wavenumber $k^{n \ell}$. Since this nonlinear propagating wavetrain is reached downstream of the source, it is denoted as $k^{n \ell+}(X, \omega)$. The forcing frequency $\omega_{f}$ and the nonlinear response wavenumber $k^{n \ell+}$ satisfy the nonlinear dispersion relation (4.4). For a more complete discussion of the relationship between linear and nonlinear spatial branches, see Pier (1999) and Pier et al. (2001).

Figure 8 illustrates the spatial response of the parallel CU wake velocity profile $(a)$ prevailing at $X=1$, at a Reynolds number $R e=100$. Forcing is applied at the origin $y_{f}=0$ with a frequency $\omega_{f}=0.19$, amplitude $A_{f}=10^{-3}$ and $\ell_{f}=1$. Total isovorticity contours and perturbation streamlines are displayed in $(b)$ and $(c)$ respectively. Close to the forcing location, the downstream response grows exponentially to reach a finite amplitude around $x \sim 70$. The measured nonlinear saturation wavenumber is $k^{n \ell+}\left(X, \omega_{f}\right)=0.27$. The solid dot at $(k, X)=(0.27,1)$ in figure $5(a)$ lies close to the contour level $\Omega^{n \ell}=\omega_{f}=0.19$, which confirms that $k^{n \ell+}=0.27$ is indeed a solution of the nonlinear dispersion relation (4.4).

The signalling problem (5.1) is numerically integrated via a finite difference scheme 
with $n_{x}$ mesh points of size $\delta x$ in the $x$-direction and $n_{y}$ Chebyshev collocation points in the $y$-direction. According to the Poisson equation $(5.1 b)$, the perturbation streamfunction $\psi$ is generated by the vorticiy field $\varpi$ which is confined within the shear region. Thus, in general, $\psi$ decays algebraically far away from the vortical region. As a result, the algebraic transformation (3.5) is still well adapted to this problem. The perturbation vorticity and streamfunction are both assumed to vanish at the upstream boundary and at infinity in the cross-stream direction. The non-reflecting boundary conditions introduced by Jin \& Braza (1993)

$$
\begin{gathered}
\frac{\partial \varpi}{\partial t}=-\left(U_{0}+\frac{\partial \psi}{\partial y}\right) \frac{\partial \varpi}{\partial x}+\frac{1}{R e} \frac{\partial^{2} \varpi}{\partial y^{2}} \\
\frac{\partial}{\partial t} \frac{\partial \psi}{\partial x}=-\left(U_{0}+\frac{\partial \psi}{\partial y}\right) \frac{\partial^{2} \psi}{\partial x^{2}}+\frac{1}{R e} \frac{\partial^{2}}{\partial y^{2}} \frac{\partial \psi}{\partial x}
\end{gathered}
$$

are implemented at the outlet. The Poisson equation $(5.1 b)$ is solved by a generalized Thomas algorithm with given boundary values of $\psi$ at the inlet and $\psi_{x}$ at the outlet. Integration in time is performed with a second-order Runge-Kutta scheme of time step $\delta t$.

The parameters chosen in the computation of figure 8 are $n_{x}=400, \delta x=0.5$, $n_{y}=31, l_{y}=5, \delta t=0.05$. The entire streamwise extent of the computational domain $-10<x<190$ is displayed to demonstrate that the outflow boundary is truly non-reflecting.

In order to analyse the spatio-temporal structure of the nonlinear response, harmonic components have been computed over one period in the stationary régime according to the Fourier expansion

$$
\psi(x, y, t)=\sum_{-\infty<n<+\infty} \phi_{(n)}(x, y) \mathrm{e}^{-\mathrm{i} n \omega_{f} t} .
$$

Figure $9(a)$ displays a snapshot of the fundamental streamwise velocity $u_{(1)}(x, y)=$ $\partial_{y} \phi_{(1)}(x, y)+$ c.c. It is seen that at the onset of nonlinearity, the fluctuation amplitude is higher than in the periodic nonlinear wavetrain reached further downstream. The associated envelope $\left|v_{(1)}\right|(x, y)=2\left|\partial_{x} \phi_{(1)}(x, y)\right|$ plotted in figure $9(b)$ effectively represents the amplitude of the fundamental cross-stream velocity fluctuations. The non-fluctuating component of the streamfunction at $n=0$ is associated with the nonlinear mean flow correction induced by Reynolds stresses. Figure $9(c)$ shows the isocontours of the mean streamwise perturbation velocity $u_{(0)}(x, y)=\partial_{y} \phi_{(0)}(x, y)$. In the finite-amplitude region, the mean flow distortion is seen to fill up the dip of the wake, thereby effectively reducing the shear experienced by the nonlinear travelling waves.

The Fourier expansion (5.5) allows the streamwise evolution of the kinetic energy $E_{n}(x)$ contained in each harmonic component to be monitored, defined as

$$
\begin{aligned}
& E_{n}(x)=\int_{-\infty}^{+\infty}\left(\left|\partial_{y} \phi_{(n)}\right|^{2}+\left|\partial_{x} \phi_{(n)}\right|^{2}\right) \mathrm{d} y \quad(n>0), \\
& E_{0}(x)=\int_{-\infty}^{+\infty} \frac{1}{2}\left(\left(\partial_{y} \phi_{(0)}\right)^{2}+\left(\partial_{x} \phi_{(0)}\right)^{2}\right) \mathrm{d} y .
\end{aligned}
$$

Semi-log plots of the streamwise evolution of $E_{n}(x)$ are given in figure 10 . In the linear region, the fundamental component (thick line) grows exponentially according to $\exp \left(-k_{i}^{\ell+} x\right)$ until a finite amplitude level is reached. The mean slope over the 

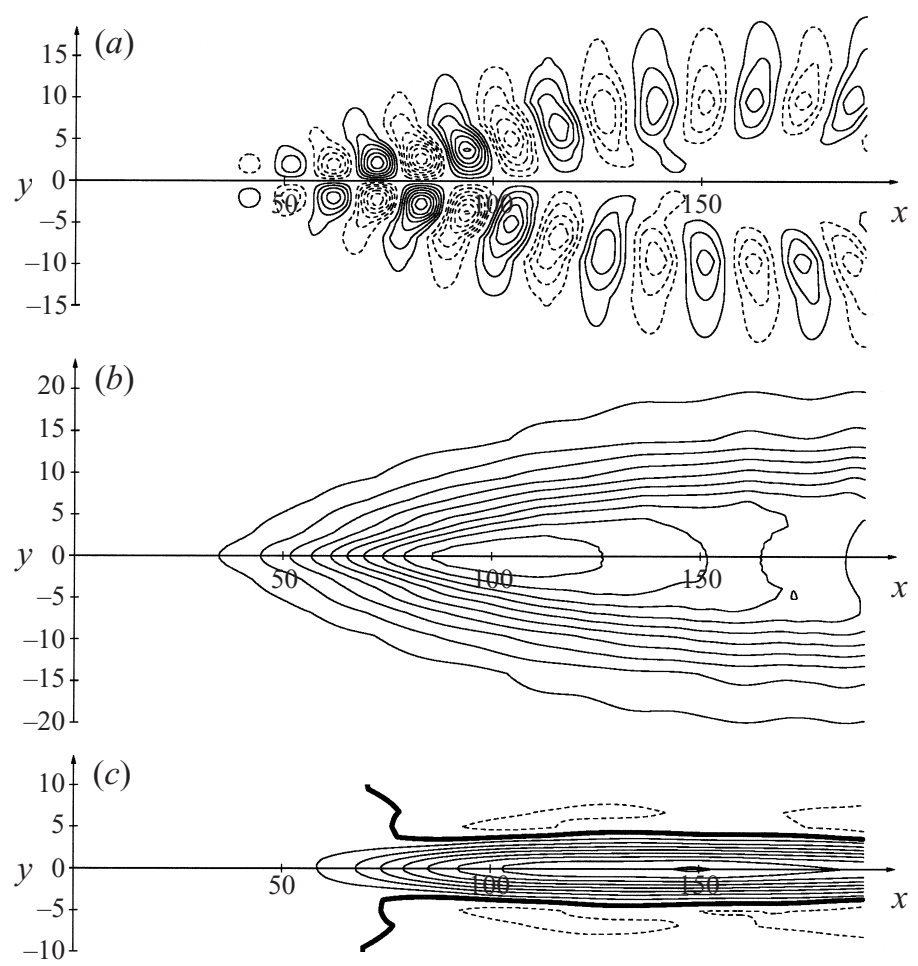

FIGURE 9. Same parallel wake spatial response as in figure 8. (a) Snapshot of fundamental streamwise velocity $u_{(1)}(x, y)$. Solid (dashed) lines pertain to $0.02,0.04, \ldots, 0.14(-0.02,-0.04, \ldots,-0.14)$ contour levels. $(b)$ Contour levels of envelope of cross-stream velocity $\left|v_{(1)}\right|(x, y)(0.02,0.04, \ldots$, $0.20)$. (c) Isolevels of nonlinear mean streamwise velocity correction $u_{(0)}(x, y)$. Thick line separates regions of positive $(0.05,0.10, \ldots, 0.40$ solid lines $)$ and negative $(-0.05$ dashed line $)$ levels.

interval $20<x<60$ is 0.054 , close to its theoretical linear value $-2 k_{i}^{\ell+} / \ln 10=0.055$ as predicted by the spatial branch $k^{\ell+}=0.316-0.064 \mathrm{i}$ computed from the OrrSommerfeld equation. Although the numerical results are not as clean as in the temporal evolution problem of figure 6 , higher harmonics are nonetheless slaved to the fundamental according to $E_{n}(x) \sim\left[E_{1}(x)\right]^{n}$. Note however that the variations of the mean-flow correction energy $E_{0}(x)$ are algebraic rather than exponential. Presumably, the vortical structures produced in the nonlinear domain generate a global mean pressure field which does not simply scale as $\left[E_{1}(x)\right]^{2}$ with the local linear spatial instability properties. This feature is absent in the temporal evolution case where the waves are strictly streamwise periodic.

With the exception of the mean flow correction, there is no feedback of the fully nonlinear downstream wavetrain on the upstream linear region. A proof of this assertion is obtained by measuring the nonlinear saturation location as a function of the forcing amplitude. The saturation station $x_{n \ell}$ may be precisely defined from the envelope of the fundamental cross-stream velocity component (see figure $9 b$ ) along the centreline $y=0$ as the location where $\left|v_{(1)}\right|(x, 0)$ reaches a preset value. The resulting variation of $x_{n \ell}$ with $-\log A_{f}$ displayed in figure 11 is clearly linear with a measured slope of 35.5 , which favourably compares with the theoretical value $-\ln 10 / k_{i}^{\ell+}=36.0$ predicted by linear theory. The nonlinear saturation station is thus solely controlled by the linear growth phase: it depends only on the forcing amplitude $A_{f}$ and linear spatial growth rate $-k_{i}^{\ell+}$. 


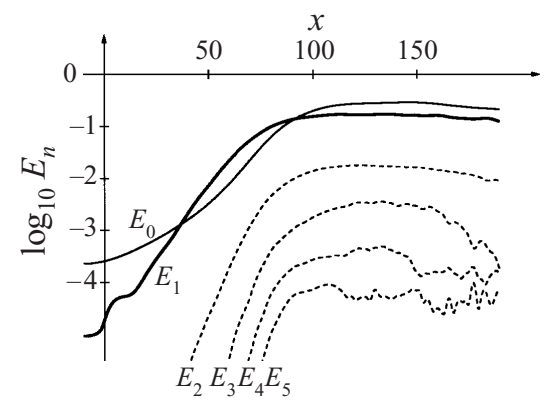

FIGURE 10. Same parallel wake spatial response as in figure 8. Semi-log plots of the streamwise evolution of the energy $E_{n}(x)$ pertaining to each harmonic component.

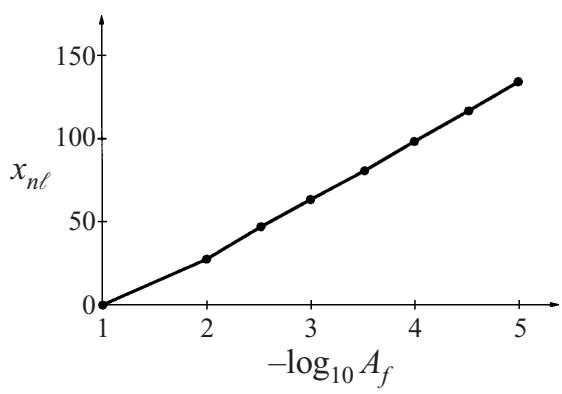

FIGURE 11. Evolution of nonlinear saturation station $x_{n \ell}$ with forcing amplitude $A_{f}$ in parallel wake. All other parameter settings same as in figure 8 .

The signalling problem (5.1) may be solved for any forcing frequency $\omega_{f}$ and any station $X$ associated with a given CU wake velocity profile. As the AU threshold at $X^{c a}$ is approached, the receptivity of the flow to forcing at the corresponding real absolute frequency $\omega_{0}^{c a}$ increases (Pier et al. 2001). At the marginal AU station $X^{c a}$, upstream and downstream spatial branches pinch, i.e. $k^{\ell+}\left(X^{c a}, \omega_{0}^{c a}\right)=k^{\ell-}\left(X^{c a}, \omega_{0}^{c a}\right)=$ $k_{0}\left(X^{c a}\right) \equiv k_{0}^{c a}$ and by definition $\Omega_{k}^{\ell}\left(k_{0}^{c a}, X^{c a}\right)=0$. Thus, according to (5.3), the spatial response diverges. In other words, the forcing amplitude required to maintain a fixed location of nonlinear saturation $x_{n \ell}$ vanishes as $\left(X, \omega_{f}\right) \rightarrow\left(X^{c a}, \omega_{0}^{c a}\right)$. In this limit, a front structure is obtained connecting a downstream fully nonlinear wavetrain to an upstream decaying tail without any forcing. This is precisely the stationary Dee-Langer front residing in the marginally AU medium.

A numerical implementation of this strategy is illustrated in figure $12(a, b)$. The forcing frequency is kept constant at $\omega_{f}=0.19 \simeq \omega_{0}^{c a}$ and the signalling problem is solved at $X=0.20$ and $X=0.22$, all other parameters being kept at the same settings. The resulting perturbation field is seen to only weakly depend on $X$ as the limit $X^{c a}=0.24$ is approached. The spatial response distribution arising from a forced problem on the CU side of $X^{c a}$ in effect provides a good approximation of the self-sustained front structure prevailing at $X^{c a}$ in the absence of forcing. The fact that resonance takes place at $X^{c a}$ is not associated with any drastic change in the perturbation field. It is only manifested by pinching of $k^{\ell+}$ and $k^{\ell-}$ branches in the complex $k$-plane.

It is naturally impractical to perform a numerical simulation at the actual $\mathrm{CU} / \mathrm{AU}$ transition since in this limit the forcing problem becomes ill-defined. However, one may attempt to verify that the spatial response would reach such a self-sustained front- 

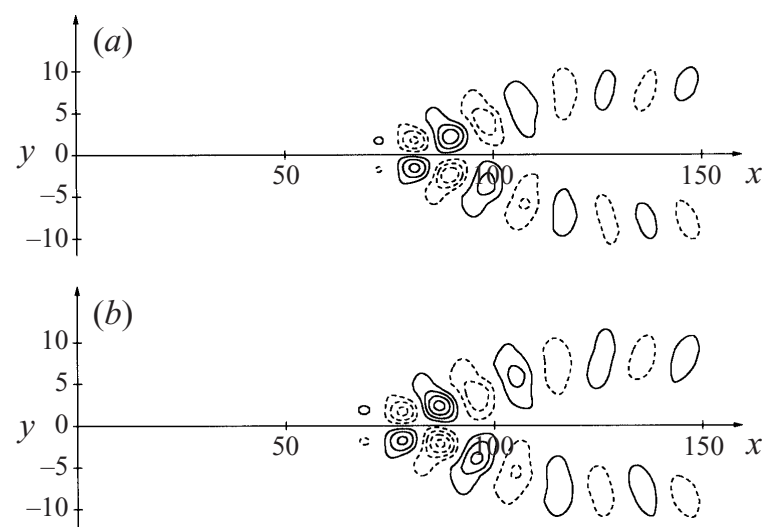

FIGURE 12. Parallel wake spatial response to forcing at frequency $\omega_{0}^{c a}=0.19$ as CU/AU transition station $X^{c a}=0.24$ is approached: isocontours of fundamental streamwise velocity at $(a) X=0.20$ (b) $X=0.22(0.05,0.10,0.15,0.20$ solid lines, $-0.05,-0.10,-0.15,-0.20$ dashed lines $)$.

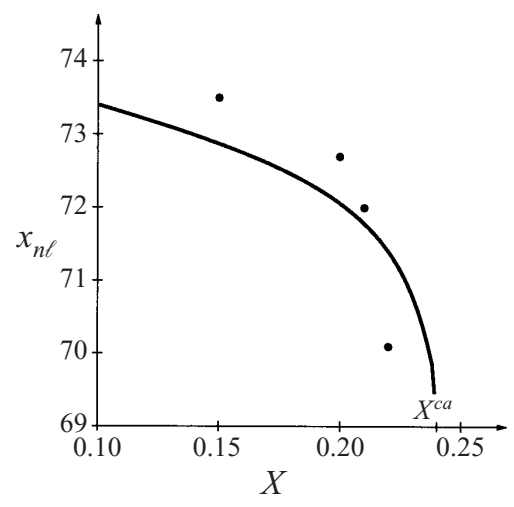

FIGURE 13. Evolution of spatial response nonlinear saturation station $x_{n \ell}$ as the marginal AU wake profile at $X^{c a}$ is approached. Comparison of numerical measurements (dots) and theoretical prediction derived from the linear dispersion relation (solid line).

like structure by tracking the nonlinear saturation station $x_{n \ell}$ as $\left(X, \omega_{f}\right) \rightarrow\left(X^{c a}, \omega_{0}^{c a}\right)$. Accordingly, the station $x_{n \ell}$ is measured for different wake velocity profiles pertaining to $X<X^{c a}=0.24$, for $\omega_{f}=\omega_{0}^{c a}=0.19$ while keeping the forcing amplitude $A_{f}$ at a fixed constant value. Results are displayed in figure 13 together with the theoretically predicted curve

$$
x_{n \ell}=\frac{\ln \left|\Omega_{k}^{\ell}\left(k^{\ell+}\left(X, \omega_{0}^{c a}\right), X\right)\right|}{-k_{i}^{\ell+}\left(X, \omega_{0}^{c a}\right)}+\text { const. }
$$

arising from the forced response (5.3). It is straightforward to demonstrate via a Taylor expansion of $\Omega_{k}^{\ell}\left(k^{\ell+}\left(X, \omega_{0}^{c a}\right), X\right)$ in the neighbourhood of $X^{c a}$ that $\left|\Omega_{k}^{\ell}\right| \propto\left|X-X^{c a}\right|^{1 / 2}$. Thus, according to (5.7), the nonlinear saturation station $x_{n \ell}$ scales as

$$
x_{n \ell} \sim \frac{1}{2} \ln \left|X-X^{c a}\right|+\text { const. } \quad \text { when } \quad X \uparrow X^{c a} .
$$

The constant appearing in the theoretical prediction (5.7) depends on the forcing amplitude $A_{f}$ and would in principle be determined by calculating the multiplicative constant in (5.3). We are solely concerned here with the functional form of $x_{n \ell}$ versus $X$ and the constant has been adjusted to achieve a reasonable fit with the numerical 
data points. Due to the slow logarithmic divergence and the insufficient accuracy of the measured $x_{n \ell}$ values, the theoretical curve only poorly fits the numerical data. This somewhat mixed result is not too surprising: the perturbation field is exponentially growing along the stream, and in order to check the validity of the scaling law (5.7), one must extract from the numerics the prefactor multiplying the exponential term. This process is numerically risky since a small error in the evaluation of the growth rate drastically contaminates the evaluation of the prefactor. It is well known that asymptotic limits are often hard to reach numerically!

\section{Self-sustained global structures in a spatially developing wake}

This part of the study is devoted to the detailed presentation of globally synchronized self-sustained structures, as obtained by direct numerical simulation of the spatially developing wake flow defined in $\S 2$. Their interpretation in terms of the previously identified elements is postponed to the next section.

Numerical simulations are performed in the vorticity/streamfunction formulation

$$
\begin{aligned}
\left(\frac{\partial}{\partial t}+U_{0}(x, y) \frac{\partial}{\partial x}\right) \varpi+U_{0, y y}(x, y) \frac{\partial \psi}{\partial x}+\left(\frac{\partial \psi}{\partial y} \frac{\partial}{\partial x}-\frac{\partial \psi}{\partial x} \frac{\partial}{\partial y}\right) \varpi & =\frac{1}{R e} \Delta \varpi, \\
\Delta \psi & =-\varpi,
\end{aligned}
$$

governing the dynamics of the perturbation fields. Note that the spatial variation of the basic flow is here expressed in terms of $x=X R e$. The following results pertain to a simulation at $R e=100$ over the domain $0<x<200(0<X<2)$. The numerical scheme exactly follows the method implemented for the signalling problem in $\S 5$ with the parallel basic flow being replaced by the spatially developing wake. Harmonic forcing is suppressed and perturbations are triggered by a small-amplitude initial impulse close to the inlet. The precise localization of the impulse is unimportant and it has been checked that the same final state is obtained for a variety of initial conditions. In the initial stage, the impulse wavepacket grows and spreads in space to perturb the entire domain. Perturbations at each streamwise station slowly synchronize and eventually lead to a global structure tuned at an overall frequency. The following results were obtained at $t=5000$, with the parameters $n_{x}=200, \delta x=1.0, n_{y}=61$, $l_{y}=6, \delta t=0.05$.

Isolines of basic and total vorticity are shown in figure $14(a, b)$, and of basic and total streamwise velocity in figure $14(c, d)$. Comparison of basic and total fields shows that the region close to the inlet is governed by small-amplitude dynamics, whereas a nonlinear wavetrain develops further downstream. In figure $14(b)$ the two vorticity layers of opposite sign are seen to be unstable and to give rise to periodic vortices which completely mask the underlying basic wake flow. The Kármán-like vortex street is more vividly illustrated by the streaklines in figure 14(e). Note that in sketches $(a-d)$ the cross-stream scale has been magnified for clarity, while $x$ - and $y$-coordinates are on the same scale in $(e)$.

Snapshots of the perturbation vorticity, $u$ - and $v$-velocity contours and streamlines are displayed in figure $15(a-d)$. The interpretation of the global mode in terms of an extended nonlinear wavepacket is illustrated by the centreline cross-stream velocity $v(x, 0, t)$ shown in figure 16 .

Vorticity time series have been recorded at different locations within the flow in order to ascertain the synchronized behaviour of the global structure. The examination of the associated frequency spectra (figure 17) indicates that all stations are tuned to 

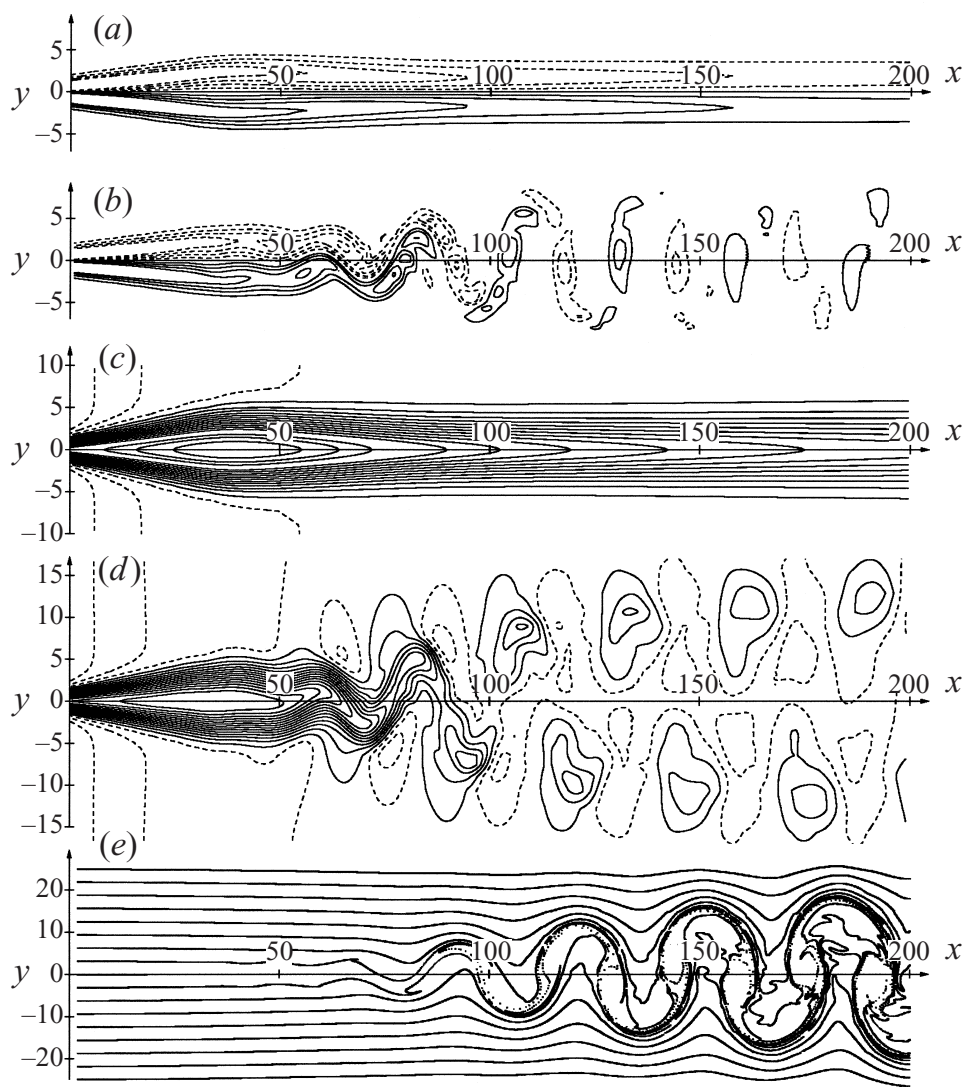

FIGURE 14. Self-sustained global structure at $R e=100$ and $t=5000$ in spatially developing wake flow defined in figure 1. $(a, b)$ Basic and total vorticity contours. Solid lines pertain to positive levels $(0.05,0.10,0.15,0.20)$, dashed lines to negative levels $(-0.05,-0.10,-0.15,-0.20)$. (c, $d)$ Basic and total streamwise velocity. Dashed lines represent the levels $0.95,0.90,0.85$ above the outlet free-stream velocity, solid lines pertain to the levels $0.80,0.75, \ldots, 0.10$. (e) Corresponding streaklines.

the same global fundamental frequency $\omega_{g}=0.186 \pm 0.002$ and its harmonics. At the station $(x, y)=(54,0)$ (figure $17 a)$ the fluctuations are seen to be almost sinusoidal. Further downstream on the centreline at $(x, y)=(150,0)$ (figure $17 c$ ) higher odd harmonics are excited. Sketch $(b)$ at $(x, y)=(114,5.6)$ shows that off the centerline all harmonics are represented. Following the analysis of Dušek, Le Gal \& Fraunié (1994) and Dušek (1996), the spatial distribution of different harmonics may be computed as displayed in figure 18. These graphs clearly demonstrate the wavetrain nature of the global mode in the downstream region. The nonlinear structures sustained by the basic wake flow give rise to a finite mean-flow distortion as shown in figure 19 . Reynolds stresses are seen to induce a mean-flow modification in the form of a pair of counter-rotating large-scale vortices (figure 19a). The associated streamwise velocity contours displayed in figure $19(b)$ reveal that the velocity defect of the basic wake profiles has been reduced by nonlinearities. The corresponding overall entrainment pattern is displayed in figure $19(c)$. Most of the dominant dynamics are captured by the fundamental and mean-flow components: superposition of the $u$-velocity contours pertaining respectively to the fundamental (figure 18a) and mean-flow distortion (figure $19 b$ ) yields a pattern which is indeed close to the total perturbation $u$-velocity 

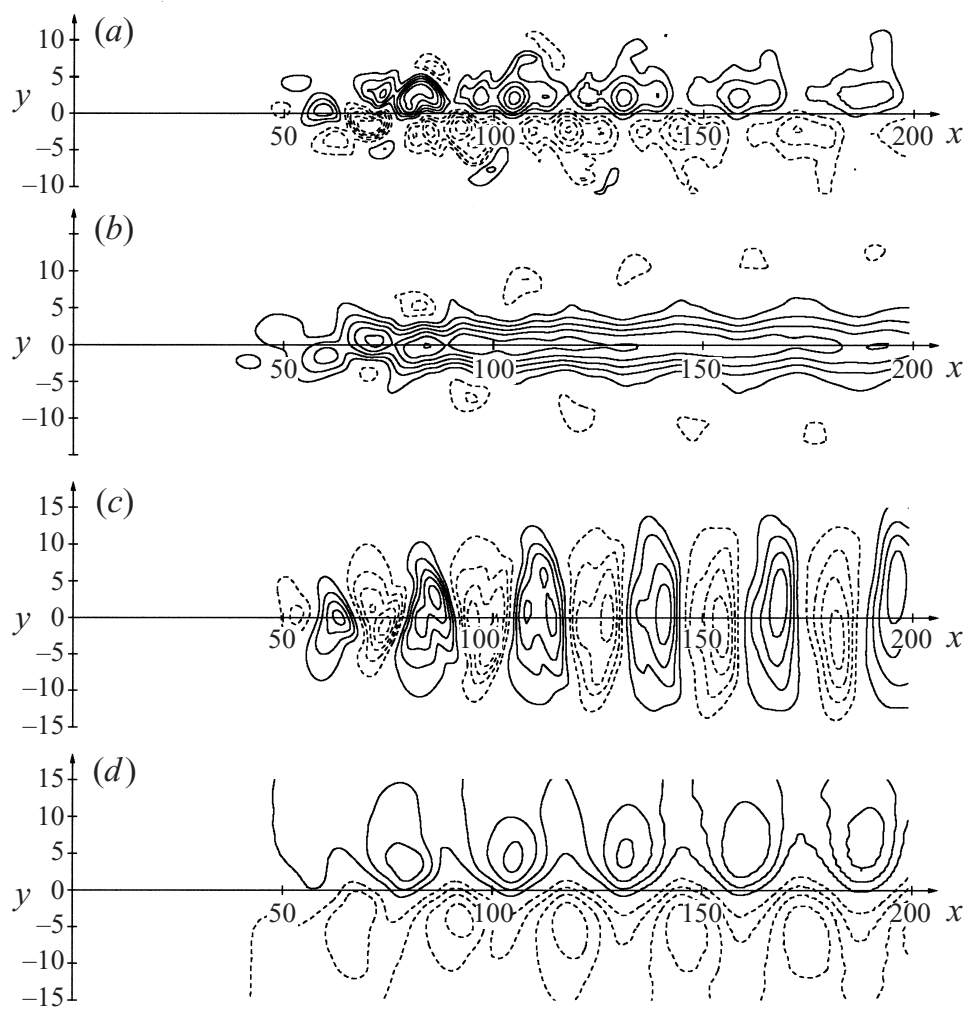

FIGURE 15. Global mode structure: instantaneous perturbation fields. Same conditions as in figure 14. (a) Isovorticity contours $(0.05,0.10, \ldots, 0.25$ solid lines, $-0.05,-0.10, \ldots,-0.25$ dashed lines). (b) Streamwise velocity contours $(0.1,0.2, \ldots, 0.6$ solid lines, $-0.1,-0.2$ dashed lines). (c) Cross-stream velocity contours $(0.05,0.10, \ldots, 0.25$ solid lines, $-0.05,-0.10, \ldots,-0.25$ dashed lines). (d) Streamlines $(0.5,1.0,1.5,2.0$ solid, $-0.5,-1.0,-1.5,-2.0$ dashed).

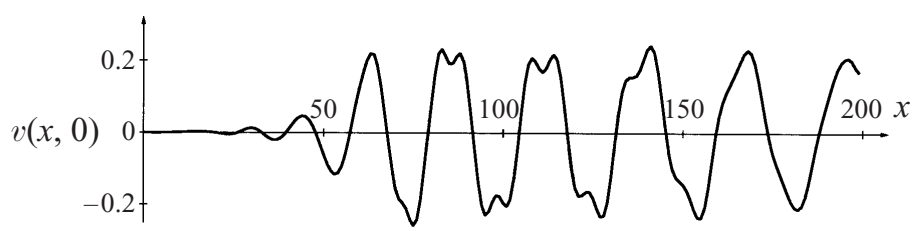

FIGURE 16. Global mode structure: instantaneous centreline cross-stream velocity $v(x, 0, t)$. Same conditions as in figure 14.

contours (figure $15 b$ ). In the same line of thought as in $\S 5$, one may display the streamwise evolution of the energy $E_{n}(x)$ contained in each harmonic (see equations (5.6)) as shown in figure 20 . Higher harmonics $(n>1)$ are seen to reach very low amplitude levels even in the nonlinear region. This observation is also corroborated by the power spectra of figure 17.

It may be concluded that the spatio-temporal development of the vortex street is essentially determined by three components: the imposed unstable basic flow, the fundamental wavetrain of frequency $\omega_{g}$ which it sustains and the ensuing mean-flow distortion produced by Reynolds stresses. 

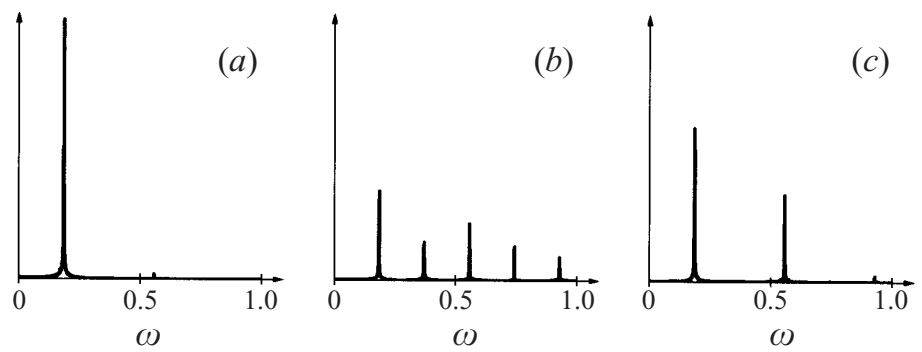

FIGURE 17. Global mode power spectrum of vorticity time series at stations $(a) x=54, y=0$, (b) $x=114, y=5.6$, (c) $x=150, y=0$, based on signal recorded over $5000<t<10000$. Same conditions as in figure 14 .
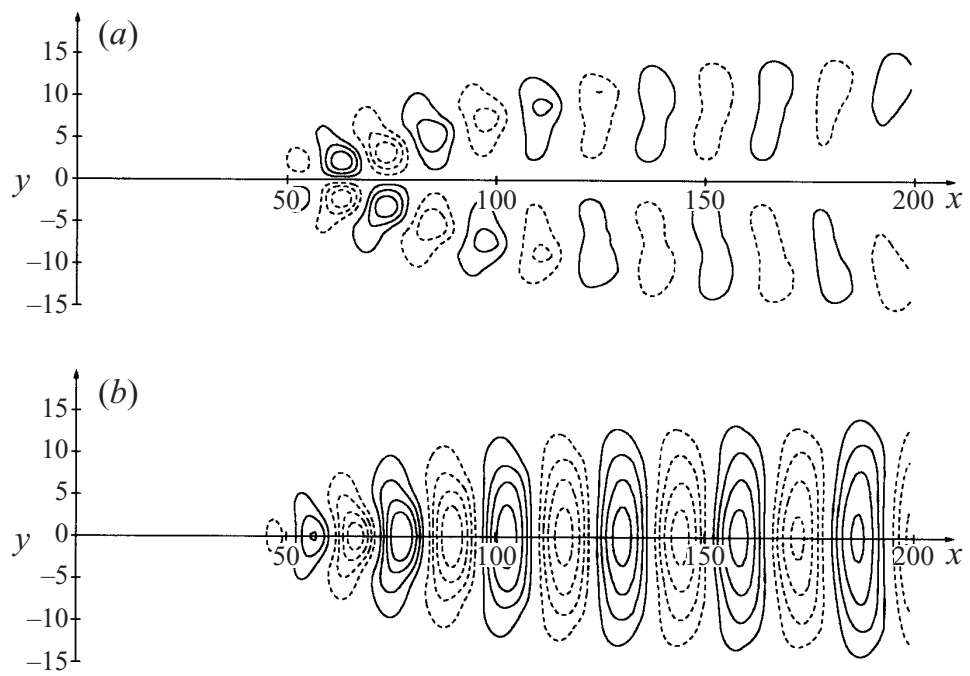

FiguRE 18. Global mode structure: fundamental velocity components. (a) Streamwise velocity contours $(0.05,0.10,0.15$ solid lines, $-0.05,-0.10,-0.15$ dashed lines). (b) Cross-stream velocity contours $(0.05,0.10,0.15,0.20$ solid lines, $-0.05,-0.10,-0.15,-0.20$ dashed lines). Same conditions as figure 14 .

\section{Interpretation of the vortex street as a steep global mode}

The objective of this section is to demonstrate that the vortex street observed in the direct numerical simulations of spatially developing wakes in $\S 6$ is indeed a steep global mode as defined by Pier et al. (1998). In other words, its frequency obeys the marginal stability criterion (1.2) and its spatio-temporal structure is controlled by a front which generates nonlinear travelling wavetrains further downstream.

The application of the steep frequency selection criterion (1.2) to the local absolute frequency $\omega_{0}(X)$ calculated in $\S 3$ and displayed in figure 3 yields

$$
X^{c a}=0.24 \text { and } \omega_{0}^{c a}=\omega_{0}\left(X^{c a}\right)=0.190,
$$

to be compared with the observed global vortex street frequency $\omega_{g}=0.186$. The prediction (7.1), which is only a leading-order approximation in $\epsilon=1 / R e=0.01$, is seen to be in excellent agreement with the value obtained by direct numerical simulation. The observed frequency should also be compared with the complex linear global frequency $\omega_{s}=0.143+0.008 \mathrm{i}$ obtained from the linear saddle-point 

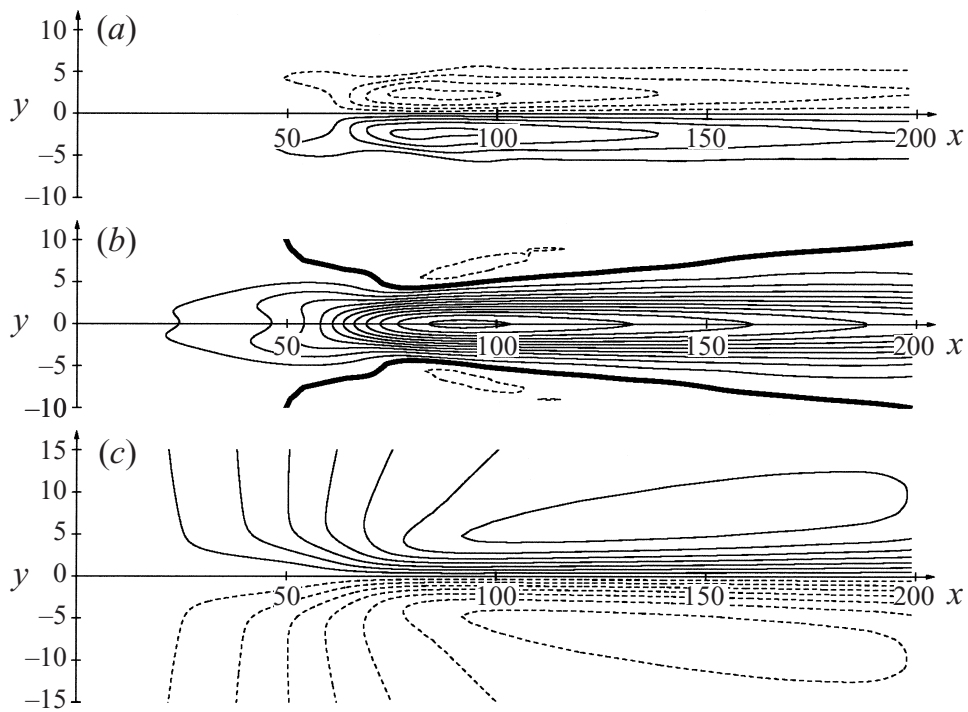

FiguRE 19. Global mode structure: finite-amplitude mean flow correction. (a) Isovorticity contours $(0.04,0.08,0.12,0.16$ solid lines, $-0.04,-0.08,-0.12,-0.16$ dashed lines $)$. (b) Streamwise velocity contours. Thick line separates regions of positive $(0.05,0.10, \ldots, 0.55$ solid lines $)$ and negative $(-0.05$ dashed line) levels. (c) Streamlines $(0.2,0.4, \ldots, 1.4$ solid lines, $-0.2,-0.4, \ldots,-1.4$ dashed lines). Same conditions as in figure 14.

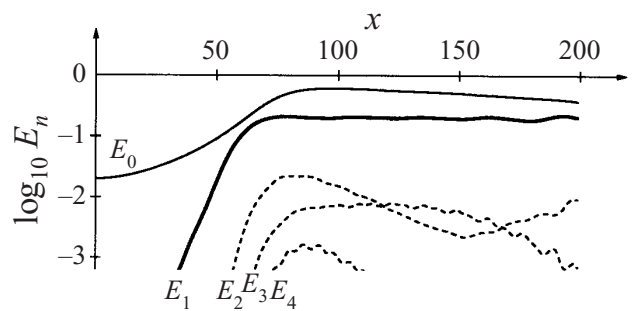

FIGURE 20. Semi-log plot of global mode spatial energy distribution of different harmonics $E_{n}(x)$. Same conditions as in figure 14.

criterion (1.1). The complex $\omega_{s}$ only applies to uniformly linear global mode structures, and does not capture the nonlinear dynamics governing the present vortex street.

The global mode spatial distribution is compared with the associated front structure and local nonlinear travelling wavetrains in figure 21. Contour levels of the fundamental streamwise velocity pertaining to the vortex street (figure $21 a$, already displayed in figure 18a) are compared with the same field for the quasi-front of frequency $\omega_{0}^{c a}=0.19$ at $X=0.22$ (figure $21 b$, already displayed in figure $12 b$ ). The pattern in figure $12(b)$ has been suitably shifted in the streamwise direction and in time so as to adjust amplitude and phase to those of figure 21(a). Although this $X$-station is below the absolute transition point $X^{c a}=0.24$, the structure of the fundamental is seen to be approximately reproduced by the front.

The same vortex street contour levels (figure 21a) are compared with those for a fully nonlinear travelling wave at $X=1.40$ obtained via temporal numerical simulation (figure 21c). More specifically, the local wavenumber $k^{n \ell+}=0.27$ prevailing at $X=1.40$ and $\omega_{0}^{c a}=0.19$ is determined from the local nonlinear dispersion relation 

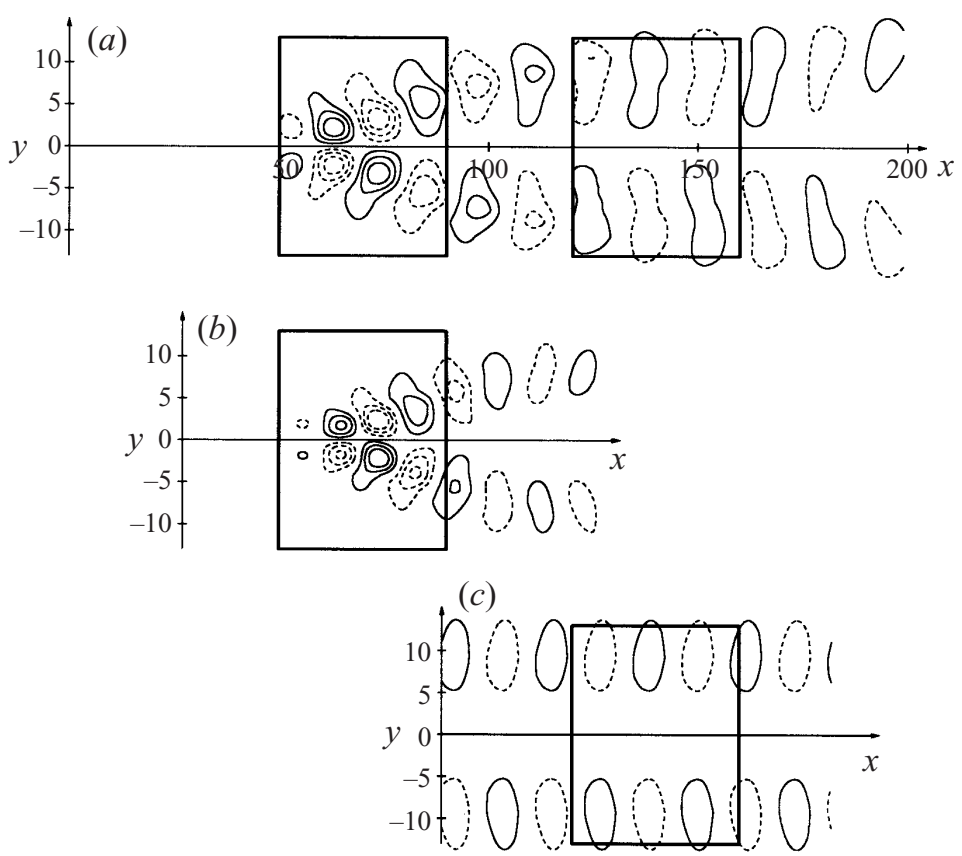

FIGURE 21. Interpretation of global mode structure $(a)$ in terms of a front $(b)$ and fully nonlinear travelling waves $(c)$. Isolevels of fundamental streamwise velocity component are shown $(0.05,0.10$, 0.15 solid lines, $-0.05,-0.10,-0.15$ dashed lines).

plotted in figure 5(a), and a streamwise periodic temporal simulation is performed as detailed in $\S 4$. Here again, the downstream region of the global mode is seen to be approximately represented by nonlinear wavetrains which travel according to the local nonlinear dispersion relation at each streamwise station.

Note however that the characteristic wavelengths of the quasi-front in $(b)$ and nonlinear travelling wave in $(c)$ appear to be somewhat smaller to those of the corresponding global mode structure in $(a)$. This feature may be related to the frequency difference between the two cases: $\omega_{0}^{c a}=0.190$ in $(b, c)$ and $\omega_{g}=0.186$ in $(a)$. The wavemaker triggering the vortex street is located at the marginally AU station $x^{c a}=\operatorname{Re} X^{c a}=24$; however, full nonlinearity is only attained about a wavelength downstream of this location. Thus the region where the global mode structure reaches finite amplitude is actually pushed to the downstream side of the AU region $24<x<55$. This result is not all that surprising: the front characterizing the streamwise transition between linear waves and a fully nonlinear wavetrain is known to typically extend over one wavelength (see e.g. van Saarloos 1987).

\section{Conclusion}

This study has demonstrated that the steep global modes previously identified and analysed in one-dimensional CGL evolution equations also arise in spatially developing shear flows exhibiting a region of absolute instability. The self-sustained structures have been shown to be controlled by a front located at the upstream edge of the AU region which acts as a wavemaker to generate nonlinear travelling waves in its wake. Provided that the streamwise variations of the underlying basic flow are sufficiently slow (WKBJ approximation), such wavetrains are governed at each 
station by the associated local nonlinear dispersion relation obtained from a purely temporal evolution problem on the local shear flow profile considered as parallel. Fast streamwise variations are confined to the front layer where the full Navier-Stokes equations have to be resolved for the wave field on the fast $x$-scale.

It should again be noted that the stationary fronts identified in the present shear flow framework are of the same nature as the propagating fronts studied in the context of pattern formation: the criterion (1.2) is merely an application of the marginal instability criterion of Dee \& Langer (1983) and van Saarloos $(1987,1988)$. The present findings are also consistent with the nature of the nonlinear impulse response in parallel wakes studied by Delbende \& Chomaz (1998). Furthermore, the conjecture put forward by Monkewitz \& Nguyen (1987) has been fully confirmed: the first streamwise station $X^{c a}$ of non-negative absolute growth rate indeed supports a front which imposes its frequency $\omega_{0}^{c a}$ on the entire flow.

The present approach constitutes a significant departure from the landmark nonlinear hydrodynamic instability analyses of spatially evolving shear flows developed by Goldstein \& Leib (1988) and Goldstein \& Hultgren (1988). These investigations require the simultaneous enforcing of the assumptions of overall small wave amplitude and slow streamwise variations. As a result, finite-amplitude effects arise only within nonlinear critical layers of small cross-stream extent. An asymptotically consistent description of the streamwise development of perturbations of given frequency may then be obtained for convectively unstable shear flows. In the present study, the small-amplitude assumption has been totally relaxed and only the slow streamwise variation hypothesis has been retained. The fully nonlinear evolution of perturbations of order unity may then be captured either for the forced problem in CU flows or for self-sustained structures in flows with an AU region. Nonlinearities then extend over the entire shear region in the cross-stream direction. Whereas critical layer studies succeed in achieving a quasi-analytic formulation of the forced dynamics, the present approach relies on local numerical simulations to identify the various constitutive elements of the dominant WKBJ approximation. It is surmised that the same fully nonlinear theory is applicable to a wide variety of spatially evolving shear flows whether they are in a self-sustained mode or subjected to forcing.

A systematic asymptotic approximation scheme of the kind presented in Pier et al. (2001) has not been attempted here. Furthermore, the Reynolds number has been kept relatively small at $R e=100$. Larger values of $R e$ increase the streamwise extent of the computational domain and computing time. Moreover, exploratory simulations indicate that secondary instabilities occur for the present basic flow beyond $R e \sim 200$. However, computation of the local nonlinear wavetrains on a streamwise periodic interval is not affected by secondary instabilities. We therefore presume that a synchronized finite-amplitude vortex shedding régime exists analytically for all large $R e$ but is unstable in the spatially developing simulation beyond $R e \sim 200$. It is thus impractical to push $R e$ up to larger values in order to move closer to the WKBJ limit $\epsilon=1 / R e=0$.

It should be emphasized that the steep frequency selection criterion (1.2) which has been demonstrated here to apply to real flows is purely based on the local linear dispersion relation, although it pertains to fully nonlinear sychronized structures. The saddle-point criterion (1.1) is also linear but it is solely applicable to strictly linear global modes. And indeed, the numerically obtained $\omega_{g}=0.186$ is much closer to $\omega_{0}^{c a}=0.190$ of criterion (1.2) than to $\omega_{s}=0.143+0.008 \mathrm{i}$ of criterion (1.1), which in any case is incompatible with the saturated nonlinear wavetrains observed downstream of the front at $X^{c a}$. Furthermore, as demonstrated by Pier (1999), steep 
global modes are triggered as soon as local linear absolute instability appears, while linear global modes become unstable only when the linear AU region is of sufficiently large extent (Chomaz et al. 1991). To confirm this argument, the same simulation has been carried out with a spatially developing wake obtained for a pressure gradient designed to yield a minimum centerline velocity of 0.047 instead of 0.010 as in figure 1 . The saddle-point frequency is then $\omega_{s}=0.155-0.001 \mathrm{i}$, which corresponds to a decaying linear global mode. Nevertheless, there is still a small locally absolutely unstable region $0.34<X<0.44$ with $\omega_{0, i}^{\max }=0.001$. In this case, a steep global mode is observed in the direct numerical simulation, whereas no self-sustained structure survives if nonlinear terms are turned off. This result is entirely consistent with the transition scenario outlined in Pier et al. (2001).

It is somewhat paradoxical that the intricate complex $X$-plane analyses developed over the years to uncover the selection properties of linear global modes are masked in practice by the onset of local absolute instability which immediately prevails and imposes its frequency and the overall structure of the synchronized oscillations.

As a final note of caution, it should be stressed that the present analysis cannot be obviously extended to bluff-body wakes where the AU region lies immediately behind the obstacle. In spite of the fact that the synthetic wake considered here is purely co-flowing, it can nonetheless sustain a synchronized vortex street which qualitatively displays the same features.

The authors are grateful to Jean-Marc Chomaz and Arnaud Couairon for enlightening discussions. The expert advice and suggestions of Carlo Cossu played an essential rôle in the design and validation of the numerical scheme.

\section{REFERENCES}

Bender, C. M. \& Orszag, S. A. 1978 Advanced Mathematical Methods for Scientists and Engineers. McGraw-Hill.

BERS, A. 1983 Space-time evolution of plasma instabilities - absolute and convective. In Handbook of Plasma Physics (ed. M. Rosenbluth \& R. Sagdeev), pp. 451-517. North-Holland.

BRIGGS, R. 1964 Electron-Stream Interaction with Plasmas. MIT Press.

Canuto, C., Hussaini, M. J., Quarteroni, A. \& Zang, T. A. 1988 Spectral Methods in Fluid Dynamics. Springer.

Chomaz, J.-M. 1992 Absolute and convective instabilities in nonlinear systems. Phys. Rev. Lett. 69, 1931-1934.

Chomaz, J.-M. \& Couairon, A. 1999 Against the wind. Phys. Fluids 11, 2977-2983.

Chomaz, J.-M., Huerre, P. \& Redekopp, L. 1991 A frequency selection criterion in spatially developing flows. Stud. Appl. Maths 84, 119-144.

Couairon, A. \& Chomaz, J.-M. 1996 Global instabilities in fully nonlinear systems. Phys. Rev. Lett. 77, 4015-4018.

Couniron, A. \& Chomaz, J.-M. 1997 a Absolute and convective instabilities, front velocities and global modes in nonlinear systems. Physica D 108, 236-276.

Couniron, A. \& Chomaz, J.-M. 1997b Pattern selection in the presence of a cross flow. Phys. Rev. Lett. 79, 2666-2669.

Couairon, A. \& Chomaz, J.-M. 1999 Fully nonlinear global modes in slowly varying flows. Phys. Fluids 11, 3688-3703.

Crighton, D. \& Gaster, M. 1976 Stability of slowly diverging jet flow. J. Fluid Mech. 77, 397-413.

Cross, M. \& Hohenberg, P. 1993 Pattern formation outside of equilibrium. Rev. Mod. Phys. 65, $851-1112$.

Dee, G. \& Langer, J. 1983 Propagating pattern selection. Phys. Rev. Lett. 50, 383-386.

Delbende, I. \& Chomaz, J.-M. 1998 Nonlinear convective/absolute instabilities in parallel twodimensional wakes. Phys. Fluids 10, 2724-2736. 
Drazin, P. G. \& ReID, W. H. 1981 Hydrodynamic Stability. Cambridge University Press.

DUŠEK, J. 1996 Spatial structure of the Bénard von Kármán instability. Eur. J. Mech. B/Fluids 15, 619-650.

Dušek, J., Le Gal, P. \& Fraunié, P. 1994 A numerical and theoretical study of the first Hopf bifurcation in a cylinder wake. J. Fluid Mech. 264, 59-80.

Ehrenstein, U. \& Koch, W. 1989 Nonlinear bifurcation study of plane Poiseuille flow. DLR Forschungsbericht 89-42. Institut für theoretische Strömungsmechanik, Göttingen.

Ehrenstein, U. \& KосH, W. 1995 Homoclinic bifurcation in Blasius boundary-layer flow. Phys. Fluids 7, 1282-1291.

Goldstein, M. \& Hultgren, L. 1988 Nonlinear spatial evolution of an externally excited instability wave in a free shear layer. J. Fluid Mech. 197, 295-330.

Goldstein, M. \& LEIB, S. 1988 Nonlinear roll-up of externally excited free shear layers. J. Fluid Mech. 191, 481-515.

Hammond, D. \& Redekopp, L. 1997 Global dynamics of symmetric and asymmetric wakes. J. Fluid Mech. 331, 231-260.

Huerre, P. \& Monkewitz, P. 1985 Absolute and convective instabilities in free shear layers. J. Fluid Mech. 159, 151-168.

Huerre, P. \& Monkewitz, P. 1990 Local and global instabilities in spatially developing flows. Ann. Rev. Fluid Mech. 22, 473-537.

Huerre, P. \& Rossi, M. 1998 Hydrodynamic instabilities in open flows. In Hydrodynamics and Nonlinear Instabilities (ed. C. Godrèche \& P. Manneville), pp. 81-294. Cambridge University Press.

Jin, G. \& BraZA, M. 1993 A nonreflecting outlet boundary condition for incompressible unsteady Navier-Stokes calculations. J. Comput. Phys. 107, 239-253.

KocH, W. 1985 Local instability characteristics and frequency determination on self-excited wake flows. J. Sound Vib. 99, 53-83.

Le Dizès, S., Huerre, P., Chomaz, J. \& Monkewitz, P. 1993 Nonlinear stability analysis of slowly-diverging flows: limitations of the weakly nonlinear approach. In Proc. IUTAM Symp. on Bluff-Body Wakes, Dynamics and Instabilities (ed. H. Eckelmann, J. Graham, P. Huerre \& P. Monkewitz), pp. 147-152. Springer.

Le Dizès, S., Huerre, P., Chomaz, J.-M. \& Monkewitz, P. 1996 Linear global modes in spatially developing media. Phil. Trans. R. Soc. Lond. A 354, 169-212.

LiNGWOOD, R. J. 1995 Absolute instability of the boundary layer on a rotating disk. J. Fluid Mech. 299, 17-33.

LingwOOD, R. J. 1996 An experimental study of absolute instability of the rotating-disk boundarylayer flow. J. Fluid Mech. 314, 373-405.

Meunier, M., Proctor, M., Sokoloff, D., Soward, A. \& Tobias, S. 1997 Asymptotic properties of a nonlinear $\alpha \omega$-dynamo wave: period, amplitude and latitude dependence. Geophys. Astrophys. Fluid Dyn. 86, 249-285.

Monkewitz, P., Bechert, D., Barsikow, B. \& Lehmann, B. 1990 Self-excited oscillations and mixing in a heated round jet. J. Fluid Mech. 213, 611-639.

Monkewitz, P., Huerre, P. \& Chomaz, J. 1993 Global linear stability analysis of weakly nonparallel shear flows. J. Fluid Mech. 251, 1-20.

Monkewitz, P. \& NGuYen, L. 1987 Absolute instability in the near-wake of two-dimensional bluff bodies. J. Fluids Struct. 1, 165-184.

PIER, B. 1999 Comportement non linéaire synchronisé dans les écoulements cisaillés. PhD thesis, École polytechnique, 91128 Palaiseau, France.

Pier, B. \& Huerre, P. 1996 Fully nonlinear global modes in spatially developing media. Physica D 97, 206-222.

Pier, B., Huerre, P. \& Chomaz, J.-M. 2001 Bifurcation to fully nonlinear synchronized structures in slowly varying media. Physica D 148, 49-96.

Pier, B., Huerre, P., Chomaz, J.-M. \& Couairon, A. 1998 Steep nonlinear global modes in spatially developing media. Phys. Fluids 10, 2433-2435.

Provansal, M., Mathis, C. \& Boyer, L. 1987 Bénard-von Kármán instability: transient and forced regimes. J. Fluid Mech. 182, 1-22.

SAARLOOS, W. VAN 1987 Dynamical velocity selection: marginal stability. Phys. Rev. Lett. 58, 25712574. 
SAARLOOS, W. VAN 1988 Front propagation into unstable states: marginal stability as a dynamical mechanism for velocity selection. Phys. Rev. A 37, 211-229.

SAARLOOS, W. VAN 1989 Front propagation into unstable states. ii. linear versus nonlinear marginal stability and rate of convergence. Phys. Rev. A 39, 6367-6390.

SAarloos, W. van \& Hohenberg, P. 1992 Fronts, pulses, sources and sinks in generalized complex Ginzburg-Landau equations. Physica D 56, 303-367.

SChär, C. \& Sмith, R. 1993 Shallow-water flow past isolated topography. Part ii: Transition to vortex shedding. J. Atmos. Sci. 50, 1404-1412.

Soward, A. 2001 Thin aspect ratio $\alpha \omega$-dynamos in galactic discs and stellar shells. In Advances in Nonlinear Dynamos (ed. M. Núñez \& A. Mas), The Fluid Mechanics of Astrophysics and Geophysics. To appear.

SowARD, A. \& JonES, C. 1983 The linear stability of the flow in the narrow gap between two concentric rotating spheres. Q. J. Mech. Appl. Math 36, 19-42.

StryKowsKi, P. \& NiCCUM, D. 1991 The stability of countercurrent mixing layers in circular jets. J. Fluid Mech. 227, 309-343.

TaYlor, M. \& Peake, N. 1999 A note on the absolute instability of wakes. Eur. J. Mech. B/Fluids 18, 573-579.

Tobias, S., Proctor, M. \& Knobloch, E. 1998 Convective and absolute instabilities of fluid flows in finite geometry. Physica D 113, 43-72.

Triantafyllou, G. \& Karniadakis, G. 1990 Computational reducibility of unsteady viscous flows. Phys. Fluids A 2, 653-656.

Triantafyllou, G., Triantafyllou, M. \& Chryssostomidis, C. 1986 On the formation of vortex streets behind stationary cylinders. J. Fluid. Mech. 170, 461-477.

Woodley, B. \& Peake, N. 1997 Global linear stability analysis of thin aerofoil wakes. J. Fluid. Mech. 339, 239-260. 